\title{
Consensus efficiency in group decision making: A comprehensive comparative study and its optimal design
}

\author{
Hengjie Zhang ${ }^{a}$, Yucheng Dong ${ }^{b, *}$, Francisco Chiclana ${ }^{c, d}$, Shui Yu ${ }^{e}$ \\ ${ }^{a}$ Business School, Hohai University, Nanjing 211100, China \\ ${ }^{b}$ Business School, Sichuan University, Chengdu 610065, China \\ ${ }^{c}$ Institute of Artificial Intelligence, De Montfort University, Leicester, UK \\ ${ }^{d}$ Dept. of Computer Science and Artificial Intelligence, University of Granada, Spain \\ ${ }^{e}$ School of Information Technology, University of Technology Sydney, Australia
}

\begin{abstract}
Consensus reaching processes (CRPs) aim to help decision-makers achieve agreement regarding the solution to a common decision problem, and consequently play an increasingly important role in the resolution of group decision making (GDM) problems. To date, a large number of CRPs have been reported. However, there is a lack of a general framework and criteria to evaluate the efficiency of the different CRPs. This paper aims to fill this gap in the research literature on CRPs. To achieve this goal, firstly, a comprehensive review regarding the different approaches to CRP is reported, and a series of CRPs as the comparison objects are presented. Secondly, the following comparison criteria for measuring the efficiency of CPRs are proposed: the number of adjusted decision-makers, the number of adjusted alternatives, the number of adjusted preference values, the distance between the original and the adjusted preference information (adjustment cost), and the number of negotiation rounds required to reach consensus. Following this, a detailed simulation experiment is designed to analyze the efficiency of different CRPs under the mentioned different comparison criteria. Furthermore, new multi-stage optimization-based CRPs are also developed, which the simulation experiment shows to have better comprehensive consensus efficiency in different GDM settings.
\end{abstract}

Keywords: Group decisions and negotiations, consensus reaching process, consensus efficiency, optimization

\section{Introduction}

Group decision making (GDM) (Cook and Kress, 1985; Greco et al., 2012; Hochbaum and Levin, 2006; Wallenius et al., 2008; Yager, 2001, 2002) can be viewed as a task to consolidate and aggregate preferences (or opinions) that a group of decision-makers express regarding a set of alternatives, which aims to find the best collective alternative solution to a decision problem. Classically, GDM models focus on how to obtain a collective solution to a decision problem without addressing whether or not consensus among decision-makers can be guaranteed. However, in many real-life contexts is crucial that all or most (a majority) of the decision-makers accept the obtained collective solution (Choudhury et al., 2006; Hartmann et al., 2009; Herrera-Viedma et al., 2002). Thus, a consensus reaching process (CRP) within the resolution of a GDM problem is usually proposed and widely implemented to help decision-makers achieve consensus regarding the obtained collective solution (Kacprzyk and Fedrizzi, 1988; Kozierkiewicz-Hetmańska, 2017). To our knowledge, it is an open problem when the GDM and consensus can yield the best solution (Arrow, 1951;

\footnotetext{
* Corresponding author.

Email addresses: hengjiezhang@hhu.edu.cn (H. Zhang), ycdong@scu.edu.cn (Y. Dong), chiclana@dmu.ac.uk (F. Chiclana), shui.yu@uts.edu.au (S. Yu).
} 
Fishburn, 1973). In relation to this, Moreno-Jiménez et al. (2013) provided an interesting discussion between efficiency and effectiveness in GDM. In spite of the fact that consensus is achieved does not necessarily imply that the best solution will be obtained, efficiency (optimal use of resources or correct decisional procedure) is a key criterion that must be met (Moreno-Jiménez et al., 2013; Roberto, 2005), which legitimizes the CRP to be a correct decision procedure with following associated key advantages (Susskind et al., 1999): (i) Building connections among the decision-makers. Using CRP as a decision tool means taking the time to find unity on how to proceed before moving forward. It is a synthesizing process that promotes the communication among decision-makers; (ii) More effective implementation. When decision-makers' preferences and concerns are considered, they are much more likely to actively participate in the implementation of the obtained solution.

The concept of consensus has been defined and studied in different ways in the literature: consensus is defined as a group opinion (Arrow, 1951), and refers to the approaches, models, tools, and procedures for deriving the final group opinion (Aguarón et al., 2016; Escobar and Moreno-Jiménez, 2007; Moreno-Jiménez et al., 2016). Moreover, several researchers defined consensus as the full and unanimous agreement of all the decision-makers regarding all the feasible alternatives (Bezdek et al., 1978), and the only consensus measurement that fits such definition to guide the CRP is the "hard" consensus based on the characteristic function with values 0 (absence consensus) and 1 (full consensus). However, unanimity may be difficult to achieve, in particular with large and diversified groups of decision-makers as is the case in real-world settings where, in addition, it may also not be necessary. In contrast, the concept of consensus has also been considered in a more flexible way with regards to its measurement, which has led to the proposal and use of "soft" consensus degrees (i.e., consensus measures) (Chiclana et al., 2013; Erdamar et al., 2014; González-Arteaga et al., 2016; Herrera-Viedma et al., 2014; Kacprzyk and Fedrizzi, 1988; Kacprzyk et al., 1997) with the aim to achieve two important goals: (i) to reflect better partial agreement; and (ii) to guide the CRP until an acceptable high level of agreement is achieved among the decision-makers. In the CRP, the soft consensus degree is often calculated by measuring similarities of preferences between individuals and the group as a whole, which is the adopted approach in this paper.

In this paper, we study the CRPs based on soft consensus. To date, a large number of CRPs based on soft consensus measures have been reported, which can be classified into the following categories (e.g., Dong et al., 2016a and 2016b): (1) CRPs with different preference representation structures; (2) CRPs based on consistency and consensus measures; (3) CRPs featuring minimum adjustments or cost; (4) CRPs in complex decision context (e.g., dynamic, Web, incomparability and incomplete contexts); (5) CRPs considering the behaviors/attitudes of decision-makers; and (6) CRPs in multiple attribute GDM (MAGDM). It should be noted that these categories are neither exhaustive nor mutually exclusive. A comprehensive review of literature regarding soft CRPs will be provided in Section 2.1.

Although a large number of CRPs have been reported in the specialized literature, there is a lack of a general framework and criteria to evaluate their efficiency. This paper aims to develop a comprehensive comparative study regarding the consensus efficiency in GDM. Specifically, the contributions to knowledge of the present research study, with the section of the paper where they are fully described indicated, are summarized below:

(1) Based on the comprehensive literature review (Section 2.1), a series of CRPs based on different consensus rules under different GDM settings are presented as the comparison objects (Section 2.2).

(2) In some applications, it is assumed that the resources for consensus building are limited and preference changing means cost. Particularly, in the last decade, the concept of minimum adjustments or minimum cost has been widely discussed in the CRP (e.g., Ben-Arieh and Easton, 2007; Ben-Arieh et al., 2009; 
Cheng et al., 2018; Dong et al., 2010a; Dong and Xu, 2016). Based on the basic idea of minimum adjustments or minimum cost, we propose the following comparison criteria for measuring the consensus efficiency of existing CRPs: the number of adjusted decision-makers; the number of adjusted alternatives; the number of adjusted preference values; the distance between the original and the adjusted preference information; and the number of negotiation rounds required to reach consensus (Section 3.1).

(3) Detailed simulation experiments are put forward for measuring the efficiency of the different CRPs under different comparison criteria (Section 3.2).

(4) Based on the results of the simulation experiments (Section 3.3), multi-stage optimization-based CRPs are designed (Section 4.1). The comprehensive consensus efficiency analysis carried out (Section 4.2) corroborates the better performance of the proposed multi-stage optimization-based CRPs in both MAGDM and GDM with preference relations (denoted as PRGDM) settings.

Finally, Section 5 discusses the advantages and limitations of our proposal, while Section 6 concludes the paper.

\section{Consensus reaching process}

In this section, we provide a comprehensive literature review on CRPs, present a series of CRPs as the comparison objects, and design detailed consensus reaching algorithms to describe them.

\subsection{Literature review on CRPs}

So far, numerous CRPs have been reported in GDM problems. In the late 1940s and early 1950s, French and his collaborators (Coch and French, 1948; French, 1956) initiated the first mathematical approaches to consensus building. In their approaches, matrix calculus was employed to model time evolution and consensus reaching. Moreover, Harary (1959), Groot (1974), and French (1981) extended consensus theory into a more general framework utilizing the Markov chain process. Afterwards, many CRPs have been proposed (e.g., Brunelli et al., 2014; Dong et al., 2017; Gong et al., 2017; Lehrer and Wagner, 1994; Martel and Kiss, 1994; Rega et al., 2006), with CRPs based on soft consensus measures, in particular, widely used because they represent better the human perception of consensus (Herrera-Viedma et al., 2014; Palomares et al., 2014a).

The CRPs based on soft consensus often involve several key aspects: preference representation, consensus measure, feedback adjustment mechanism, decision context, and behaviors of decision-makers. Regarding the main aspects of focus in the CRP study, we list different types of CRPs based on soft consensus reported in the literature. Notably, a CRP study may focus on multiple combinations of the aspects introduced above, so the listed categories of CRPs based on soft consensus are not mutually exhaustive.

(1) CRPs with different preference representation structures. In GDM, preference representation structures (e.g., utility functions (Tanino, 1990), preference orderings (Dong and Zhang, 2014), and preference relations (Herrera et al., 1997; Kacprzyk et al., 1992; Orlovsky, 1978; Saaty, 1980)) are effective tools for decision-makers to express their preferences on a set of alternatives. In the current literature, many CRPs with different preference representation structures have been reported. For instance, Kacprzyk et al. (1992) proposed a CRP for GDM with additive preference relations, while Cabrerizo et al. (2010) reported a CRP for GDM with the incomplete linguistic preference relations based on unbalanced linguistic term sets. Furthermore, Herrera-Viedma et al. (2002), Choudhury et al. (2006) and Dong and Zhang (2014) presented CRPs that address heterogeneous preference information.

(2) CRPs based on consistency and consensus measures. In GDM problems with preference relations (e.g., additive, multiplicative, and linguistic preference relations), individual consistency plays a key role in 
ensuring that decision-makers are neither random nor illogical in providing their preferences. However, in searching group consensus the CRP may destroy individual consistency. To maintain the individual consistency in the consensus building, CRPs based on individual consistency and consensus measures have been proposed. For example, Herrera et al. (1997), Chiclana et al. (2008), Zhang and Guo (2016), and $\mathrm{Wu}$ and $\mathrm{Xu}$ (2016b) proposed two-stage based consensus frameworks for integrating individual consistency measure, while Dong et al. (2010b, 2015b), Zhang et al. (2014), and Wu and Xu (2012) developed consensus frameworks that simultaneously manage individual consistency and consensus. Meanwhile, Moreno-Jiménez et al. (2005, 2008) and Escobar et al. (2015) developed consistency consensus matrix based approaches to manage the individual consistency and consensus in AHP-GDM problems in an elegant and concise way.

(3) CRPs featuring minimum adjustments or cost. An important issue in building consensus is the design of an effective feedback process. In particular, several CRPs with minimum adjustments or cost have been investigated. Dong et al. (2010a) proposed a CRP with minimum adjustments in a linguistic context. Meanwhile, Ben-Arieh and Easton (2007) presented a CRP with minimum cost, which they subsequently extended to a maximum decision-maker CRP (Ben-Arieh and Easton, 2009). Also, Zhang et al. (2011) investigated the minimum cost CRP by taking aggregation functions into account; Dong et al. (2015a) and Dong and Xu (2016) presented a CRP with minimum adjustments in a hesitant linguistic GDM context and in different GDM contexts, respectively. In addition, the CRP with minimum cost has been pursued by Gong et al. (2015a, 2015b) and, recently, by Zhang et al. (2017a) with a 2-rank CRP with minimum adjustments for multi-granular linguistic GDM.

(4) CRPs in complex GDM (e.g., dynamic GDM, Web-based GDM, GDM with incomparability or incomplete preference information). Societal and technological trends demand the management of CRPs in complex GDM problems (Pérez et al., 2018). To address these complex GDM problems,, Pérez et al. (2010) proposed a dynamic CRP to manage decision situations in which the set of alternatives changes dynamically; Alonso et al. (2010) and Kacprzyk and Zadrozny (2010a) investigated web-based consensus support systems; Dong et al. (2016a) reported a CRP for complex and dynamic GDM framework; Parreiras et al. (2012a) proposed a dynamic CRP with incomparability fuzzy preference relations; Zhang et al. (2018) developed a CRP for the GDM with incomplete linguistic distribution assessments.

(5) CRPs considering the behaviors/attitudes of decision-makers. In addition to mathematical aspects, real-world consensus building also involves the psychological behaviors of decision-makers. Recently, several CRPs that consider the behaviors/attitudes of decision-makers have been reported in the literature. Altuzarra et al. (2010) investigated the negotiation attitudes of the decision-makers and put forward a number of semiautomatic initiatives for establishing consensus in the AHP-GDM problem. Palomares et al. (2014c), Quesada et al. (2015), Xu et al. (2015a), and Dong et al. (2016b) all have studied the CRP by addressing non-cooperative behaviors of the decision-makers, while Wu and Chiclana (2015) have proposed a trust-based CRP.

(6) CRPs in MAGDM. In some GDM problems, decision-makers evaluate alternatives based on multiple attributes (e.g., Greco et al., 2014; Maier and Stix, 2013), which is known as MAGDM problems. As in the case of GDM, many CRPs have also been proposed for MAGDM problems. Guha and Chakraborty (2011) investigated consensus in MAGDM by implementing the degrees of confidence of decision-makers' preferences. Moreover, Parreiras et al. (2010) put forward a flexible CRP for MAGDM with linguistic assessments, and $\mathrm{Xu}$ et al. (2015b) proposed a consensus approach for eliminating conflicts in emergency MAGDM problems. 
The above six categories of CRPs based on soft consensus measurement are summarized in Table 1.

Table 1: A summary of different CRPs based on soft consensus

\begin{tabular}{|c|c|c|}
\hline CRPs category & Literature & $\begin{array}{l}\text { Number of } \\
\text { publications }\end{array}$ \\
\hline $\begin{array}{l}\text { CRPs with different } \\
\text { preference representation } \\
\text { structures }\end{array}$ & $\begin{array}{l}\text { Altuzarra et al., 2010; Ben-Arieh and Chen, 2006; Cabrerizo et al., } \\
2009 \text { and 2010; Chen et al., 2015; Choudhury et al., 2006; Cook, 2006; } \\
\text { Dong and Zhang, 2014; Escobar et al., 2015; Escobar and } \\
\text { Moreno-Jiménez, 2007; Fedrizzi et al., 1988; Herrera-Viedma et al., } \\
\text { 2002, 2005 and 2007a; Herrera et al., 1997; Kacprzyk and Fedrizzi, } \\
\text { 1988; Kacprzyk et al., 1992, } 1997 \text { and 2010b; Mata et al., 2009; } \\
\text { Moreno-Jiménez et al., 2016; Pedrycz and Song, 2011; Srdjevic et al., } \\
\text { 2013; Szmidt and Kacprzyk, 2003; Xu and Wu, 2013 }\end{array}$ & 25 \\
\hline $\begin{array}{l}\text { CRPs based on consistency } \\
\text { and consensus measures }\end{array}$ & $\begin{array}{l}\text { Aguarón et al., 2016; Chiclana et al., 2008; Dong et al., 2010b and } \\
\text { 2015b; Escobar et al. 2015; Herrera et al., 1997; Meng et al., 2016; } \\
\text { Moreno-Jiménez et al., } 2005 \text { and 2008; Wu and Xu, } 2012 \text { and 2016b; } \\
\text { Zhang et al., } 2014\end{array}$ & 12 \\
\hline $\begin{array}{l}\text { CRPs featuring minimum } \\
\text { adjustments or cost }\end{array}$ & $\begin{array}{l}\text { Ben-Arieh and Easton, 2007; Ben-Arieh et al., 2009; Cheng et al., } \\
\text { 2018; Dong et al., 2010a, 2015a and 2015b; Dong and Xu, 2016; Gong } \\
\text { et al., 2015a, 2015b and 2015c; Lee, 2002; Zhang et al., } 2011 \text { and } \\
\text { 2017a }\end{array}$ & 13 \\
\hline CRPs in complex GDM & $\begin{array}{l}\text { Alonso et al., } 2010 \text { and 2013; Cao et al., 2008; Dong and Cooper, } \\
\text { 2016; Dong et al., 2016a; Fedrizzi et al., 1999; Kacprzyk and } \\
\text { Zadrożny, 2010a; Lawryshyn et al., 2017; Parreiras et al., 2012a; Pérez } \\
\text { et al., } 2010 \text { and 2018; Zhang et al., } 2018\end{array}$ & 12 \\
\hline $\begin{array}{l}\text { CRPs considering the } \\
\text { behaviors or attitudes of } \\
\text { decision-makers }\end{array}$ & $\begin{array}{l}\text { Altuzarra et al., 2010; Dong et al., 2016b; Hartmann et al., 2009; Li et } \\
\text { al., 2017; Palomares et al., 2014c; Pérez et al., 2014; Quesada et al., } \\
\text { 2015; Rega et al., 2006; Wu et al., } 2015 \text { and 2016; Xu et al., 2015a }\end{array}$ & 11 \\
\hline CRPs in MAGDM & $\begin{array}{l}\text { Dong et al., 2016a; Guha et al., 2011; Kim et al., 1999; Ma et al., 2014; } \\
\text { Parreiras et al., } 2010 \text { and 2012b; Sun and Ma, 2015; Wu and Xu, } \\
\text { 2016a; Xu et al., } 2014 \text { and 2015a; Yu and Lai, 2011; Zhang et al., } \\
\text { 2017a }\end{array}$ & 12 \\
\hline
\end{tabular}

Based on the above literature review, we notice that despite the great achievements made in the CRP research area, there is still a lack of a general framework and criteria to compare the efficiency of different CRPs. Moreover, we find that different CRPs, which were proposed for different GDM problems, share some common characteristics:

(1) Consensus rule. Preference-modification in the feedback adjustment process of the CRPs is often driven by the implementation of two kinds of consensus rules:

(i) Identification Rule (IR) and Direction rule (DR) (e.g., Herrera-Viedma et al., 2002, 2014). The first one is used to identify the decision-makers, alternatives and preference values contributing less to consensus and that, consequently, should be considered in the feedback adjustment process; while the second one provides the direction of the preference-modification to increase the group consensus.

(ii) Optimization-based consensus rules (e.g., Dong et al., 2010a; Dong and Xu, 2016) to minimize the number of decision-makers, alternatives and preference values to be considered in the feedback adjustment process, as well as the distance between the original and the adjusted preferences. For brevity, we will use "adjusted decision-makers, alternatives and preference values" when referring to the decision-makers, alternatives and preference values to be considered in the feedback adjustment process. 
(2) Decision pattern. Existing CRPs are often investigated under two basic decision patterns: MAGDM and PRGDM. In MAGDM, decision-makers provide their preferences on a set of alternatives regarding a set of attributes, and the preference representation format is the multiple attribute decision matrix. In PRGDM, decision-makers provide their preferences on all pairs of alternatives, and the preference representation format is the preference relation (also called pairwise comparison matrix).

\subsection{Comparison objects}

Based on the different consensus rules and different GDM patterns introduced in Section 2.1, this section presents a series of CRPs as the comparison objects.

(1) CRPs in the MAGDM setting

(i) MAGDM problem. The MAGDM problem can be described as follows:

There are three basic elements in MAGDM: a finite set of alternatives, $X=\left\{x_{1}, x_{2}, \ldots, x_{n}\right\} \quad(n \geq 2)$; a finite set of decision-makers, $E=\left\{e_{1}, e_{2}, \ldots, e_{m}\right\} \quad(m \geq 2)$; and a finite set of attributes $A=\left\{a_{1}, a_{2}, \ldots, a_{l}\right\} \quad(l \geq 2)$. For simplicity, let $M=\{1,2, \ldots, m\}, N=\{1,2, \ldots, n\}$, and $L=\{1,2, \ldots, l\}$. Let $\lambda=\left(\lambda_{1}, \lambda_{2}, \ldots, \lambda_{m}\right)^{T}$ be the decision-makers weighting vector, where $\lambda_{k} \geq 0$ is the weight associated to the decision-maker $e_{k}$ and $\sum_{k=1}^{m} \lambda_{k}=1$. Let $w=\left(w_{1}, w_{2}, \ldots, w_{l}\right)^{T}$ be the attributes weighting vector, where $w_{j} \geq 0$ is the weight associated to the attribute $a_{j}$ and $\sum_{j=1}^{l} w_{j}=1$. Let $V^{(k)}=\left(v_{i j}^{(k)}\right)_{n \times l}$ be the multiple attribute decision matrix provided by the decision-maker $e_{k}$, where $v_{i j}^{(k)}$ represents his/her preference value for the alternative $x_{i}$ with respect to the attribute $a_{j}$. Without loss of generality, we assume that $v_{i j}^{(k)} \in[0,1]$. The decision objective of the MAGDM is to obtain a ranking of the alternatives, from best to worst, based on the multiple attribute decision matrices $V^{(k)}=\left(v_{i j}^{(k)}\right)_{n \times l} \quad(k \in M)$.

(ii) Consensus measures in MAGDM. In GDM, aggregation operators are often implemented to fuse the individual preferences, with the Weighted Average (WA) operator and Ordered Weighted Average (OWA) operator (Yager, 1988) being frequently used in the different models reviewed in Section 2.1. In any case, the use of a particular aggregation operator will not influence the findings of this paper and therefore, without loss of generality, the WA operator will be employed in this paper to compute the collective multiple attribute decision matrix, $V^{(c)}=\left(v_{i j}^{(c)}\right)_{n \times l}$ :

$$
v_{i j}^{(c)}=\sum_{k=1}^{m} \lambda_{k} \cdot v_{i j}^{(k)}
$$

Let $C D\left(e_{k}\right)$ be the consensus degree of the decision-maker $e_{k} ; C D\left(x_{i}\right)$ the consensus degree of the alternative $x_{i} ; C D\left(v_{i j}\right)$ the consensus degree of the preference value $v_{i j}$; and $C D\left\{e_{1}, e_{2}, \ldots, e_{m}\right\}$ the consensus degree among all the decision-makers $\left\{e_{1}, e_{2}, \ldots, e_{m}\right\}$, computed using the following expressions (Wu and Xu, 2016a):

$$
\begin{gathered}
C D\left(e_{k}\right)=1-\frac{1}{n \times l} \sum_{i=1}^{n} \sum_{j=1}^{l}\left|v_{i j}^{(k)}-v_{i j}^{(c)}\right| \\
C D\left(x_{i}\right)=1-\frac{1}{m \times l} \sum_{k=1}^{m} \sum_{j=1}^{l}\left|v_{i j}^{(k)}-v_{i j}^{(c)}\right| \\
C D\left(v_{i j}\right)=1-\frac{1}{m} \sum_{k=1}^{m}\left|v_{i j}^{(k)}-v_{i j}^{(c)}\right|
\end{gathered}
$$




$$
C D\left\{e_{1}, e_{2}, \ldots, e_{m}\right\}=1-\frac{1}{m \times n \times l} \sum_{k=1}^{m} \sum_{i=1}^{n} \sum_{j=1}^{l}\left|v_{i j}^{(k)}-v_{i j}^{(c)}\right|
$$

Notice that the above consensus degrees all are in the unit interval $[0,1]$. The maximum value 1 indicates full consensus with respect to the collective preference; and the higher the value the higher the consensus degree. For example, all decision-makers fully agree with the collective preference when $C D\left\{e_{1}, e_{2}, \ldots, e_{m}\right\}=1$, and the larger the $C D\left\{e_{1}, e_{2}, \ldots, e_{m}\right\}$ value the higher the consensus degree among the set of decision-makers.

(iii) The design of CRPs in the MAGDM setting. For convenience, the CRP in the MAGDM setting is denoted as MACRP. We use IR.E, IR.A and IR.P to denote that the rule to identify the decision-makers, alternatives and preference values contributing less to consensus, respectively. Moreover, when the optimization-based consensus rule is utilized to minimize the number of adjusted decision-makers, alternatives and preference values, and the distance between the original and the adjusted preferences, the following notation is adopted: OE, OA, OP, OD, respectively. Taking the different consensus rules into account the following eight MACRPs are identified and briefly described in Table 2.

Table 2: MACRPs

\begin{tabular}{|c|c|c|}
\hline MACRPs & $\begin{array}{c}\text { Consensus } \\
\text { rules }\end{array}$ & Brief description \\
\hline MACRP 1 & IR.E-DR & $\begin{array}{l}\text { In each consensus round of MACRP } 1 \text {, IR.E is used to identify the decision-maker with the } \\
\text { lowest consensus degree, and DR finds out the direction for the identified decision-maker to } \\
\text { modify his/her decision matrix. }\end{array}$ \\
\hline MACRP 2 & IR.A-DR & $\begin{array}{l}\text { In each consensus round of MACRP } 2 \text {, IR.A is used to identify the alternative with the } \\
\text { lowest consensus degree, and DR is used to help decision-makers modify their preferences } \\
\text { regarding the identified alternative. }\end{array}$ \\
\hline MACRP 3 & IR.P-DR & $\begin{array}{l}\text { In each consensus round of MACRP } 3 \text {, IR.P is used to identify the preference value with the } \\
\text { lowest consensus degree, and DR is used to help decision-makers modify their preferences } \\
\text { regarding the identified preference values. }\end{array}$ \\
\hline MACRP 4 & DR & $\begin{array}{l}\text { In each consensus round of MACRP } 4, \text { DR helps decision-makers find out the direction to } \\
\text { change their decision matrices. }\end{array}$ \\
\hline MACRP 5 & $\mathrm{OE}$ & $\begin{array}{l}\text { In each consensus round of MACRP } 5 \text {, the optimization consensus rule is used to obtain the } \\
\text { optimal adjusted decision matrices by minimizing the number of adjusted decision-makers, } \\
\text { and which are used as the references for decision-makers to modify their decision matrices. }\end{array}$ \\
\hline MACRP 6 & $\mathrm{OA}$ & $\begin{array}{l}\text { In each consensus round of MACRP } 6 \text {, the optimization consensus rule is used to obtain the } \\
\text { optimal adjusted decision matrices by minimizing the number of adjusted alternatives, and } \\
\text { which are used as the references decision-makers to modify their decision matrices. }\end{array}$ \\
\hline MACRP 7 & OP & $\begin{array}{l}\text { In each consensus round of MACRP } 7 \text {, the optimization consensus rule is used to obtain the } \\
\text { optimal adjusted decision matrices by minimizing the number of adjusted preference values, } \\
\text { and which are used as the references for decision-makers to modify their decision matrices. }\end{array}$ \\
\hline MACRP 8 & OD & $\begin{array}{l}\text { In each consensus round of MACRP } 8 \text {, the optimization consensus rule is used to obtain the } \\
\text { optimal adjusted decision matrices by minimizing the distance between individual original } \\
\text { and adjusted decision matrices, and which are used as the references for decision-makers to } \\
\text { modify their decision matrices. }\end{array}$ \\
\hline
\end{tabular}

The eight MACRPs are formally presented below.

(a) MACRP 1. In each consensus round of MACRP 1, according to IR.E, the decision-maker $e_{o}$ contributing less to consensus are identified, i.e. $C D\left(e_{o}\right)=\min _{k} C D\left(e_{k}\right)$. Thus, decision-maker $e_{o}$ should modify his/her preference according to DR, while the other decision-makers' preferences remain unchanged. 
Let $\overline{V^{(k)}}=\left(\overline{v_{i j}^{(k)}}\right)_{n \times l}$ be the adjusted multiple attribute decision matrix associated with $V^{(k)}=\left(v_{i j}^{(k)}\right)_{n \times l}$. Specifically, the following is used by decision-makers to modify their decision matrices: a) for $k=o$, $i \in N$, and $j \in L$, let $\overline{v_{i j}^{(k)}} \in\left[\min \left(v_{i j}^{(k)}, v_{i j}^{(c)}\right), \max \left(v_{i j}^{(k)}, v_{i j}^{(c)}\right)\right]$; b) for $k \in M, k \neq O, i \in N$, and $j \in L$, then let $\overline{v_{i j}^{(k)}}=v_{i j}^{(k)}$.

Here, we put forward Algorithm M-1 to describe MACRP 1, which is provided in Appendix A.

(b) MACRP 2. In each consensus round of MACRP 2, IR.A is activated to identify the alternative $x_{p}$ with the lowest consensus degree, i.e., $C D\left(x_{p}\right)=\min _{i} C D\left(x_{i}\right)$. As a result, the preference information regarding alternative $x_{p}$ should be modified, while other alternatives' preference information remain unchanged. Then, according to DR, decision-makers modify their preferences as follows: a) for $k \in M$, $i=p$, and $j \in L$, let $\overline{v_{i j}^{(k)}} \in\left[\min \left(v_{i j}^{(k)}, v_{i j}^{(c)}\right), \max \left(v_{i j}^{(k)}, v_{i j}^{(c)}\right)\right]$; b) for $k \in M, i \in N, i \neq p$, and $j \in L$, let $\overline{v_{i j}^{(k)}}=v_{i j}^{(k)}$.

Algorithm M-2 to describe MACRP 2 is Algorithm M-1 with Step 3 replaced with Step 3-A below:

Step 3-A: Let $C D_{z}\left(x_{p}\right)=\min _{i} C D_{z}\left(x_{i}\right)$. We recommend decision-makers to update multiple attribute decision matrices $V_{z+1}^{(k)}=\left(v_{i j, z+1}^{(k)}\right)_{n \times l}$ as follows: a) for $k \in M, i=p$, and $j \in L$, let $v_{i j, z+1}^{(k)} \in\left[\min \left(v_{i j, z}^{(k)}, v_{i j, z}^{(c)}\right), \max \left(v_{i j, z}^{(k)}, v_{i j, z}^{(c)}\right)\right]$; b) for $k \in M, i \in N, i \neq p$, and $j \in L$, let $v_{i j, z+1}^{(k)}=v_{i j, z}^{(k)}$.

(c) MACRP 3. In each consensus round of MACRP 3, the preference value $v_{p q}$ with the lowest consensus degree is identified using IR.P, i.e., $C D\left(v_{p q}\right)=\min _{i, j} C D\left(v_{i j}\right)$. Therefore, the preference information regarding the preference value $v_{p q}$ should be modified, while the rest of preference values remain unchanged. Based on DR, decision-makers modify their multiple attribute decision matrices as follows: a) for $k \in M, i=p$, and $j=q$, let $\overline{v_{i j}^{(k)}} \in\left[\min \left(v_{i j}^{(k)}, v_{i j}^{(c)}\right), \quad \max \left(v_{i j}^{(k)}, v_{i j}^{(c)}\right)\right]$; b) for $k \in M, i \in N, i \neq p$, $j \in L$, and $j \neq q$, let $\overline{v_{i j}^{(k)}}=v_{i j}^{(k)}$.

Algorithm M-3 to describe MACRP 3 is Algorithm M-1 with Step 3 replaced with Step 3-B below:

Step 3-B: Let $C D\left(v_{r s}\right)=\min _{i, j} C D\left(v_{i j}\right)$. Decision-makers are advised to update their respective multiple attribute decision matrices $V_{z+1}^{(k)}=\left(v_{i j, z+1}^{(k)}\right)_{n \times l}$ as follows: a) for $k \in M, i=p$, and $j=q$, let $v_{i j, z+1}^{(k)} \in\left[\min \left(v_{i j, z}^{(k)}, v_{i j, z}^{(c)}\right), \max \left(v_{i j, z}^{(k)}, v_{i j, z}^{(c)}\right)\right]$; b) for $k \in M, i \in N, i \neq p, j \in L$, and $j \neq q$, let $v_{i j, z+1}^{(k)}=v_{i j, z}^{(k)}$.

(d) MACRP 4. In MACRP 4, DR is used to help decision-makers provide their multiple attribute decision matrices $\overline{V^{(k)}}=\left(\overline{v_{i j}^{(k)}}\right)_{n \times l}$ : for $k \in M, i \in N$, and $j \in L$, let $\overline{v_{i j}^{(k)}} \in\left[\min \left(v_{i j}^{(k)}, v_{i j}^{(c)}\right), \max \left(v_{i j}^{(k)}, v_{i j}^{(c)}\right)\right]$.

Algorithm M-3 to describe MACRP 4 is Algorithm M-1 with Step 3 replaced with Step 3-C below:

Step 3-C: Update multiple attribute decision matrices $V_{z+1}^{(k)}=\left(v_{i j, z+1}^{(k)}\right)_{n \times l}$ as follows: for $k \in M, i \in N$, and $j \in L$, let $v_{i j, z+1}^{(k)} \in\left[\min \left(v_{i j, z}^{(k)}, v_{i j, z}^{(c)}\right), \max \left(v_{i j, z}^{(k)}, v_{i j, z}^{(c)}\right)\right]$.

(e) MACRP 5. In the CRP, the number of adjusted decision-makers should be as small as possible, which is achieved by implementing the following optimization-based consensus rule:

$$
\min \sum_{k=1}^{m} z^{(k)}
$$

where 


$$
z^{(k)}=\left\{\begin{array}{ll}
0, & v_{i j}^{(k)}=\overline{v_{i j}^{(k)}}, \forall i, j \\
1, & \text { otherwise }
\end{array} .\right.
$$

Meanwhile, it is natural that the consensus degree among decision-makers should be acceptable, i.e., $C D\left\{e_{1}, e_{2}, \ldots, e_{m}\right\}=1-\frac{1}{m \times n \times l} \sum_{k=1}^{m} \overline{v_{i j}^{(k)}}-\overline{v_{i j}^{(c)}} \mid \geq \alpha$. Thus, the following optimization-based consensus model to minimize the number of adjusted decision-makers is constructed:

$$
\begin{aligned}
& \min \sum_{k=1}^{m} z^{(k)} \\
& \text { s.t. }\left\{\begin{array}{l}
1-\frac{1}{m \times n \times l} \sum_{k=1}^{m} \sum_{i=1}^{n} \sum_{j=1}^{l}\left|\overline{v_{i j}^{(k)}}-\overline{v_{i j}^{(c)}}\right| \geq \alpha \\
v_{i j}^{(c)}=\sum_{k=1}^{m} \lambda_{k} \cdot \overline{v_{i j}^{(k)}}, i \in N ; j \in L \\
z^{(k)}=\left\{\begin{array}{ll}
0, & v_{i j}^{(k)}=\overline{v_{i j}^{(k)}} \\
1, & \text { otherwise }
\end{array}, \forall i, j\right.
\end{array} .\right.
\end{aligned}
$$

In model (8), $\overline{V^{(k)}}=\left(\overline{v_{i j}^{(k)}}\right)_{n \times l}$ and $z^{(k)} \quad(k \in M)$ are decision variables. To easily solve model (8), it is transformed into a mixed 0-1 linear programming model (given in Appendix B). Solving model (8) yields the optimal solution to $z^{(k)}, z^{(k, *)}(k \in M)$, which results in the optimal solution to $\overline{V^{(k)}}=\left(\overline{v_{i j}^{(k)}}\right)_{n \times l}$, $V^{(k, *)}=\left(v_{i j}^{(k, *)}\right)_{n \times l} \quad(k \in M)$ and the corresponding MACRP 5 can be designed. The core idea of MACRP 5 is to return $V^{\left(k,{ }^{*}\right)}=\left(v_{i j}^{(k, *)}\right)_{n \times l} \quad(k \in M)$ to decision-maker $e_{k}$ to reconsider constructing a new multiple attribute decision matrix $\overline{V^{(k)}}=\left(\overline{v_{i j}^{(k)}}\right)_{n \times l}$. When constructing $\overline{V^{(k)}}=\left(\overline{v_{i j}^{(k)}}\right)_{n \times l}$, it is advised that $\overline{v_{i j}^{(k)}} \in\left[\min \left(v_{i j}^{(k)}, v_{i j}^{(k, *)}\right), \max \left(v_{i j}^{(k)}, v_{i j}^{(c, *)}\right)\right]$.

It should be emphasized that in CRPs with optimization-based consensus rules, the optimal adjusted preference information obtained by the optimization-based consensus models are used as a reference for the preferences-modifying in the CRPs. To ensure the convergence of the CRPs, we can use the more stringent consensus standard in the optimization-based consensus models than in the CRPs.

Algorithm M-5 to describe MACRP 5 is Algorithm M-1 with Step 3 replaced with Step 3-D below:

Step 3-D: The optimal multiple attribute decision matrices $V_{z}^{(k, *)}=\left(v_{i j, z}^{(k, *)}\right)_{n \times l}(k \in M)$ can be yielded by solving model (8). We provide $V_{z}^{(k, *)}=\left(v_{i j, z}^{(k, *)}\right)_{n \times l}(k \in M)$ as the references for decision-makers to express their updated multiple attribute decision matrices $V_{z+1}^{(k)}=\left(v_{i j, z+1}^{(k)}\right)_{n \times l}$ using the following: for $k \in M$, $i \in N$, and $j \in L$, let $v_{i j, z+1}^{(k)} \in\left[\min \left(v_{i j, z}^{(k)}, v_{i j, z}^{(k, *)}\right), \max \left(v_{i j, z}^{(k)}, v_{i j, z}^{(k, *)}\right)\right]$.

(f) MACRP 6. Naturally, the number of adjusted alternatives should be as small as possible in the CRPs. Following this idea, the following model is used to minimize the number of adjusted alternatives:

$$
\min \sum_{k=1}^{m} \sum_{i=1}^{n} y_{i}^{(k)}
$$

where,

$$
y_{i}^{(k)}= \begin{cases}0, & v_{i j}^{(k)}=\overline{v_{i j}^{(k)}}, \forall j \\ 1, & \text { otherwise }\end{cases}
$$

Meanwhile, the consensus degree among decision-makers should be acceptable, that is 
$C D\left\{e_{1}, e_{2}, \ldots, e_{m}\right\} \geq \alpha$. Therefore, the following optimization-based consensus model is presented to support MACRP 6:

$$
\begin{aligned}
& \min \sum_{k=1}^{m} \sum_{i=1}^{n} y_{i}^{(k)} \\
& \text { s.t. }\left\{\begin{array}{l}
1-\frac{1}{m \times n \times l} \sum_{k=1}^{m} \sum_{i=1}^{n} \sum_{j=1}^{l}\left|\overline{v_{i j}^{(k)}}-\overline{v_{i j}^{(c)}}\right| \geq \alpha \\
\overline{v_{i j}^{(c)}}=\sum_{k=1}^{m} \lambda_{k} \cdot \overline{v_{i j}^{(k)}}, i \in N ; j \in L \\
y_{i}^{(k)}= \begin{cases}0, & v_{i j}^{(k)}=\overline{v_{i j}^{(k)}}, \forall j \\
1, & \text { otherwise }\end{cases}
\end{array}\right.
\end{aligned}
$$

Clearly, in model (11), $\overline{V^{(k)}}=\left(\overline{v_{i j}^{(k)}}\right)_{n \times l}$ and $y_{i}^{(k)}(k \in M, i \in N)$ are decision variables. Solving model (11) involves its transformation into a mixed 0-1 linear programming model (given in Appendix C). The optimal solution to $y_{i}^{(k)}$ is denoted as $y_{i}^{(k, *)}(k \in M, i \in N)$, while the optimal solution to $\overline{V^{(k)}}=\left(\overline{v_{i j}^{(k)}}\right)_{n \times l}$ is denoted as $V^{\left(k, k^{*}\right)}=\left(v_{i j}^{(k, *)}\right)_{n \times l} \quad(k \in M)$, and MACRP 6 described with Algorithm M-6 by replacing Eq. (8) in Step 3-D of Algorithm M-5 with Eq. (11) to become a new Step 3-E.

(g) MACRP 7. In CRPs, the number of adjusted preference values should be as small as possible. To do this, the optimization-based consensus rule that minimizes the adjusted preference values is adopted, i.e.,

$$
\min \sum_{k=1}^{m} \sum_{i=1}^{n} \sum_{j=1}^{l} x_{i j}^{(k)}
$$

where

$$
x_{i j}^{(k)}=\left\{\begin{array}{ll}
0, & v_{i j}^{(k)}=\overline{v_{i j}^{(k)}} \\
1, & \text { otherwise }
\end{array} .\right.
$$

Meanwhile, the consensus degree among decision-makers is acceptable, i.e., $C D\left\{e_{1}, e_{2}, \ldots, e_{m}\right\} \geq \alpha$. Based on this, the following optimization-based consensus model is proposed:

$$
\begin{aligned}
& \min \sum_{k=1}^{m} \sum_{i=1}^{n} \sum_{j=1}^{l} x_{i j}^{(k)} \\
& \text { s.t. }\left\{\begin{array}{l}
1-\frac{1}{m \times n \times l} \sum_{k=1}^{m} \sum_{i=1}^{n} \sum_{j=1}^{l} \overline{\mid v_{i j}^{(k)}}-\overline{v_{i j}^{(c)}} \mid \geq \alpha \\
v_{i j}^{(c)}=\sum_{k=1}^{m} \lambda_{k} \cdot \overline{v_{i j}^{(k)}}, i \in N ; j \in L \\
x_{i j}^{(k)}= \begin{cases}0, & v_{i j}^{(k)}=\overline{v_{i j}^{(k)}} \\
1, & v_{i j}^{(k)} \neq \overline{v_{i j}^{(k)}}\end{cases}
\end{array}\right.
\end{aligned}
$$

It is clear that $\overline{V^{(k)}}=\left(\overline{v_{i j}^{(k)}}\right)_{n \times l}$ and $x_{i j}^{(k)} \quad(k \in M, i \in N, j \in L)$ are decision variables in model (14). Solving this model involves its transformation into a mixed 0-1 linear programming model (given in Appendix D). We use $x_{i j}^{(k, *)}$ to denote the optimal solution with respect to $x_{i j}^{(k)} \quad(k \in M, i \in N, j \in L)$, and $V^{(k, *)}=\left(v_{i j}^{(k, *)}\right)_{n \times l}$ for the optimal solution associated with $\overline{V^{(k)}}=\left(\overline{v_{i j}^{(k)}}\right)_{n \times l} \quad(k \in M, i \in N, j \in L)$. Similarly to MACRP 5, MACRP 7 is described with Algorithm M-7, which is generated by replacing Eq. (8) into Eq. (14) in Step 3-D of Algorithm M-5 to generate Step 3-F. 
(h) MACRP 8. In the CRP, the distance between individual original and adjusted decision matrices should be as small as possible, that is to find the solution to

$$
\min \sum_{k=1}^{m} d\left(V^{(k)}, \overline{V^{(k)}}\right)
$$

where $d\left(V^{(k)}, \overline{V^{(k)}}\right)=\sum_{k=1}^{m} \sum_{i=1}^{n} \sum_{j=1}^{l}\left|v_{i j}^{(k)}-\overline{v_{i j}^{(k)}}\right|$ denotes the distance between $V^{(k)}$ and $\overline{V^{(k)}}$. Meanwhile, the predefined consensus degree should be achieved among decision-makers, i.e., $C D\left\{e_{1}, e_{2}, \ldots, e_{m}\right\} \geq \alpha$. Based on this, the following optimization-based consensus is put forward:

$$
\begin{aligned}
& \min \sum_{k=1}^{m} \sum_{i=1}^{n} \sum_{j=1}^{l}\left|v_{i j}^{(k)}-\overline{v_{i j}^{(k)}}\right| \\
& \text { s.t. }\left\{\begin{array}{l}
1-\frac{1}{m \times n \times l} \sum_{k=1}^{m} \sum_{i=1}^{n} \sum_{j=1}^{l}\left|\overline{v_{i j}^{(k)}}-\overline{v_{i j}^{(c)}}\right| \geq \alpha . \\
\overline{v_{i j}^{(c)}}=\sum_{k=1}^{m} \lambda_{k} \cdot \overline{v_{i j}^{(k)}}, i \in N ; j \in L
\end{array}\right.
\end{aligned}
$$

$\overline{V^{(k)}}=\left(\overline{v_{i j}^{(k)}}\right)_{n \times l} \quad(k \in M)$ are decision variables in model (16). This model can be transformed into a linear programming model (given in Appendix E) with optimal solution $V^{(k, *)}=\left(v_{i j}^{(k, *)}\right)_{n \times l} \quad(k \in M, i \in N, j \in L)$. MACRP 8 is described using Algorithm M-8, with is obtained from Algorithm M-5 by replacing Eq. (8) into Eq. (16) in Step 3-D to derive Step 3-G.

\section{(2) CRPs in the PRGDM setting}

(i) PRGDM problem. As mentioned before, preference relations are widely used as the representation format of decision-makers' preferences. The different types of preference relations in literature include: multiplicative preference relations (Fedrizzi and Giove, 2013; Saaty, 1980; Srdjevic, 2007), fuzzy preference relations (Herrera-Viedma et al., 2007b; Palomares et al., 2014a), and linguistic preference relations (Dong et al., 2015b; Herrera et al., 1997). Chen et al. (2015) reviewed the transformation functions between the different formats of preference relations. In this paper, we assume that decision-makers provide their preferences on the set of alternatives by means of additive fuzzy preference relations (also called additive preference relations). Thus, representing by $P^{(k)}=\left(p_{i j}^{(k)}\right)_{n \times n}(k \in M)$ the additive fuzzy preference relation of decision-maker $e_{k}$, where $p_{i j}^{(k)} \in[0,1]$ represents his/her preference degree on the alternative $x_{i}$ over $x_{j}$, it is assumed the following additive reciprocity property: $p_{i j}^{(k)}+p_{j i}^{(k)}=1 \quad \forall i, j \in N$. The decision objective of the PRGDM is to obtain a ranking of alternatives, from best to worst, based on a set of additive preference relations $P^{(k)}=\left(p_{i j}^{(k)}\right)_{n \times n} ;(k \in M)$. For simplicity, in what follows, we will refer to additive fuzzy preference relations as simply preference relations.

(ii) Consensus measures in PRGDM. Let $P^{(c)}=\left(p_{i j}^{(c)}\right)_{n \times n}$ be the collective preference relation

$$
p_{i j}^{(c)}=\sum_{k=1}^{m} \lambda_{k} \cdot p_{i j}^{(k)}
$$

Let $C D\left(p_{i j}\right)$ be the consensus degree of the preference value $p_{i j}$ and $C D\left(e_{k}\right), C D\left(x_{i}\right)$, and $C D\left\{e_{1}, e_{2}, \ldots, e_{m}\right\}$ as described before for the MAGDM case. Under the PRGDM context, their respective expressions are (Cabrerizo et al., 2010; Chiclana et al., 2013): 


$$
\begin{gathered}
C D\left(e_{k}\right)=1-\frac{1}{n \times(n-1)} \sum_{i=1}^{n} \sum_{j=1, j \neq i}^{n}\left|p_{i j}^{(k)}-p_{i j}^{(c)}\right| \\
C D\left(x_{i}\right)=1-\frac{1}{m \times(n-1)} \sum_{i=1}^{n} \sum_{j=1, j \neq i}^{n}\left|p_{i j}^{(k)}-p_{i j}^{(c)}\right| \\
C D\left(p_{i j}\right)=1-\frac{1}{m} \sum_{k=1}^{m}\left|p_{i j}^{(k)}-p_{i j}^{(c)}\right| \\
C D\left\{e_{1}, e_{2}, \ldots, e_{m}\right\}=1-\frac{1}{m \times n \times(n-1)} \sum_{k=1}^{m} \sum_{i=1}^{n} \sum_{j=1, j \neq i}^{n}\left|p_{i j}^{(k)}-p_{i j}^{(c)}\right|
\end{gathered}
$$

(iii) The design of CRPs in the PRGDM setting. For convenience, the CRP under the PRGDM setting

\begin{tabular}{|c|c|c|}
\hline PRCRP & $\begin{array}{l}\text { Consensus } \\
\text { rule }\end{array}$ & Brief description \\
\hline PRCRP 1 & IR.E-DR & $\begin{array}{l}\text { In each round of PRCRP } 1 \text {, IR.E is used to identify the decision-maker with the lowest } \\
\text { consensus degree, and DR is used to find out the direction for the identified decision-maker } \\
\text { to modify his/her preference relation. }\end{array}$ \\
\hline PRCRP 2 & IR.A-DR & $\begin{array}{l}\text { In each consensus round of PRCRP } 2 \text {, IR.A is used to identify the alternative with the } \\
\text { lowest consensus degree, and DR is used to help decision-makers modify their preferences } \\
\text { regarding the identified alternative. }\end{array}$ \\
\hline PRCRP 3 & IR.P-DR & $\begin{array}{l}\text { In each consensus round of PRCRP 3, IR.P is used to identify the preference value with the } \\
\text { lowest consensus degree, and DR is used to help decision-makers modify their preferences } \\
\text { regarding the identified preference values. }\end{array}$ \\
\hline PRCRP 4 & DR & $\begin{array}{l}\text { In each consensus round of PRCRP } 4 \text {, DR helps decision-makers find out the direction to } \\
\text { change their preference relations. }\end{array}$ \\
\hline PRCRP 5 & $\mathrm{OE}$ & $\begin{array}{l}\text { In each consensus round of PRCRP 5, the optimization-based consensus rule is adopted to } \\
\text { obtain the optimal adjusted preference relations by minimizing the adjusted } \\
\text { decision-makers, and which are used as the references for decision-makers to modify their } \\
\text { preference relations. }\end{array}$ \\
\hline PRCRP 6 & OA & $\begin{array}{l}\text { In each consensus round of PRCRP } 6 \text {, the optimization-based consensus rule is utilized to } \\
\text { yield the optimal adjusted preference relations by minimizing the adjusted alternatives, and } \\
\text { which are used as the references for decision-makers to modify their preference relations. }\end{array}$ \\
\hline PRCRP 7 & OP & $\begin{array}{l}\text { In each consensus round of PRCRP } 7 \text {, the optimization-based consensus rule is used to } \\
\text { yield the optimal adjusted preference relations by minimizing the number of adjusted } \\
\text { preference values, and which are used as the references for decision-makers to modify their } \\
\text { preference relations. }\end{array}$ \\
\hline PRCRP 8 & OD & $\begin{array}{l}\text { In each consensus round of PRCRP } 8 \text {, the optimization-based consensus rule is adopted to } \\
\text { generate the optimal adjusted preference relations by minimizing the distance between } \\
\text { individual original and adjusted preference relations, and which are used as the references } \\
\text { for decision-makers to modify their preference relations. }\end{array}$ \\
\hline
\end{tabular}
is denoted as PRCRP. By taking the different consensus rules into account in the PRCRPs, the following eight PRCRPs are identified and briefly described in Table 3.

Table 3: PRCRPs

Models and Algorithms can be designed for PRCRPs 1-8 in a similar way as to the MACRPs 1-8 described before, respectively. The main difference between MACRPs and PRCRPs being that decision-makers take different preference information formats; multiple attribute decision matrices are used to represent preferences in MACRPs, while preference relations are used in PRCRPs. Due to space limitation, we can safely omit the detailed development of Models and Algorithms for PRCRPs 1-8.

\section{Comparison analysis}

In this section, we present several comparison criteria for measuring consensus efficiency, and we 
design simulation experiment to evaluate the efficiency of CRPs in different decision contexts.

\subsection{Comparison criteria}

In what follows, it is assumed that a preference change has associated a cost and that the resources for consensus building are limited. Following the basic idea of minimum adjustments (e.g., Dong et al., 2010a; Dong and $\mathrm{Xu}, 2016$ ), we propose the following criteria to compare the efficiency of CRPs: number of adjusted decision-makers, number of adjusted alternatives, number of adjusted preference values, and the distance between the original and the adjusted preference information. Additionally, the number of consensus rounds required to reach consensus is also considered a criterion to measure the consensus efficiency. The proposed comparison criteria are introduced below.

Let $D^{(k)}=\left(d_{i j}^{(k)}\right)_{p \times q}$ be the preference information (multiple attribute decision matrices or preference relations) associated with decision-makers $e_{k}(k=1,2, \ldots, m)$. Let $\overline{D^{(k)}}=\left(\overline{d_{i j}^{(k)}}\right)_{p \times q}$ be the adjusted preference information associated with $D^{(k)}=\left(d_{i j}^{(k)}\right)_{p \times q}(k=1,2, \ldots, m)$. Let $D=\left\{D^{(1)}, D^{(2)}, \ldots, D^{(m)}\right\}$ and $\bar{D}=\left\{\overline{D^{(1)}}, \overline{D^{(2)}}, \ldots, \overline{D^{(m)}}\right\}$.

(1) The number of adjusted decision-makers. Let $z^{(k)}$ be a $0-1$ variable. If the preference information of decision-maker $e_{k}$ does not change, then $z^{(k)}=0$; otherwise $z^{(k)}=1$, i.e.

$$
z^{(k)}=\left\{\begin{array}{ll}
0, & d_{i j}^{(k)}=\overline{d_{i j}^{(k)}}, \forall i, j \\
1, & \text { otherwise }
\end{array} .\right.
$$

The total number of adjusted decision-makers is:

$$
A E=\sum_{k=1}^{m} z^{(k)}
$$

Clearly, $A E \geq 0$, with $A E=0$ indicating that the decision-makers' preference information remains unchanged. The smaller the value of $A E$ the better the efficiency of the CRP is.

(2) The number of adjusted alternatives. Let $y_{i}^{(k)}$ be a 0-1 variable. If the preference information of alternative $x_{i}$ does not change, then $y_{i}^{(k)}=0$; otherwise $y_{i}^{(k)}=1$; i.e.

$$
y_{i}^{(k)}=\left\{\begin{array}{ll}
0, & d_{i j}^{(k)}=\overline{d_{i j}^{(k)}}, \forall j \\
1, & \text { otherwise }
\end{array} .\right.
$$

The total number of adjusted alternatives is:

$$
A A=\sum_{k=1}^{m} \sum_{i=1}^{p} y_{i}^{(k)} .
$$

It is clear that $A A \geq 0$, with $A A=0$ representing that the decision-makers' preference information does not change. The smaller the value of $A A$ the better the efficiency of the CRP is.

(3) The number of adjusted preference values. Let $x_{i j}^{(k)}$ be a 0-1 variable. If the preference information of the preference value $d_{i j}$ does not change, then $x_{i j}^{(k)}=0$; otherwise $x_{i j}^{(k)}=1$, i.e.

$$
x_{i j}^{(k)}=\left\{\begin{array}{ll}
0, & d_{i j}^{(k)}=\overline{d_{i j}^{(k)}} \\
1, & \text { otherwise }
\end{array} .\right.
$$

The total number of adjusted preference values is:

$$
A P=\sum_{k=1}^{m} \sum_{i=1}^{p} \sum_{j=1}^{q} x_{i j}^{(k)}
$$

Again, we have that $A P \geq 0$, with $A P=0$ meaning that the decision-makers' preferences remain unchanged. The smaller the value of $A P$ the better the efficiency of the consensus model is. 
(4) The distance between the original and the adjusted preference information. There are many mathematical functions available to measure the distance between real numbers. In GDM, the Manhattan distance has been widely used to measure the distance between preferences, and as such it is adopted here as well: $\quad d\left(D^{(k)}, \overline{D^{(k)}}\right)=\sum_{i=1}^{p} \sum_{j=1}^{q}\left|d_{i j}^{(k)}-\overline{d_{i j}^{(k)}}\right|$. The overall change between $D=\left\{D^{(1)}, D^{(2)}, \ldots, D^{(m)}\right\} \quad$ and $\bar{D}=\left\{\overline{D^{(1)}}, \overline{D^{(2)}}, \ldots, \overline{D^{(m)}}\right\}$ is:

$$
d(D, \bar{D})=\sum_{k=1}^{m} \sum_{i=1}^{p} \sum_{j=1}^{q}\left|d_{i j}^{(k)}-\overline{d_{i j}^{(k)}}\right| .
$$

It is $d(D, \bar{D}) \geq 0$, with $d(D, \bar{D})=0$ meaning that the decision-makers' preference information does not change. The smaller the value of $d(D, \bar{D})$ the better the efficiency of the CRP is.

(5) The iterations to reach consensus. In a CRP, reaching consensus requires a number of negotiation rounds (denoted here by $Z$ ). The number of iterations required to reach a predefined consensus degree can be considered as a criterion to measure the efficiency of a CRP, especially for emergency decision-making problems that need to reach consensus as fast as possible.

Note 1: As mentioned before, the existing CRPs share some common characteristics: consensus rules and basic decision patterns (i.e., MAGDM and PRGDM). The proposed comparison criteria are based on the common characteristics in CPRs, and are useful to provide a basic framework to evaluate the performance of CRPs. However, when studying the CRPs in some complex decision contexts, we need to introduce more metrics to evaluate their performance based on specific decision problems. For example, when considering the category of CRPs based on consistency and consensus measures, it becomes evident the necessity of a metric to evaluate their performance in achieving consistent preference relations (Li et al., 2018).

\subsection{Simulation experiments design}

Let $A E_{t}, A A_{t}, A P_{t}, A D_{t}$ and $Z_{t}$ be the number of adjusted decision-makers, the number of adjusted alternatives, the number of adjusted preference values, the distance between the original and adjusted preference information, and the number of consensus rounds, in MACRP $t$ (PRCRP $t$ ) $(t=1,2, \ldots, 8)$, respectively. This section presents simulation experiments to compare the consensus efficiency of the eight different MACRPs (MACRPs 1-8) and PRCRPs (PRCRPs 1-8) by randomly and uniformly generating the preference information of decision-makers.

(1) The simulation method for measuring the efficiency of MACRPs. Simulation method I, as described in Table 4, is designed to evaluate the efficiency of the eight different MACRPs.

Table 4: Simulation method I

Input: $m, n, l, \alpha, \lambda$

Output: $A E_{t}, A A_{t}, A P_{t}, A D_{t}$ and $Z_{t}(t=1,2, \ldots, 8)$

Step 1: Generate $m$ decision matrices $V^{(k)}=\left(v_{i j}^{(k)}\right)_{n \times l}(k \in M)$, where $v_{i j}^{(k)}$ is randomly and uniformly generated from interval $[0,1]$.

Step 2: Take $\left\{V^{(k)}=\left(v_{i j}^{(k)}\right)_{n \times l}(k=1,2, \ldots, m), \alpha, \lambda\right\}$ as the inputs of MACRP $t(t=1,2, \ldots, 8)$; obtain the adjusted decision matrices, $\overline{V^{(k, t)}}=\left(\overline{v_{i j}^{(k, t)}}\right)_{n \times l}(k \in M)$, and the number of consensus rounds, $Z_{t}$ $(t=1,2, \ldots, 8)$.

Step 3: Based on $V^{(k)}=\left(v_{i j}^{(k)}\right)_{n \times l}$ and $\overline{V^{(k, t)}}=\left(\overline{v_{i j}^{(k, t)}}\right)_{n \times l} \quad(k \in M)(t=1,2, \ldots, 8)$ obtain $A E_{t}, A A_{t}$, $A P_{t}$, and $A D_{t}$, respectively.

(2) The simulation method for measuring the efficiency of PRCRPs. Simulation method II, as described in Table 5, is designed to assess the efficiency of the eight different PRCRPs.

Table 5: Simulation method II 


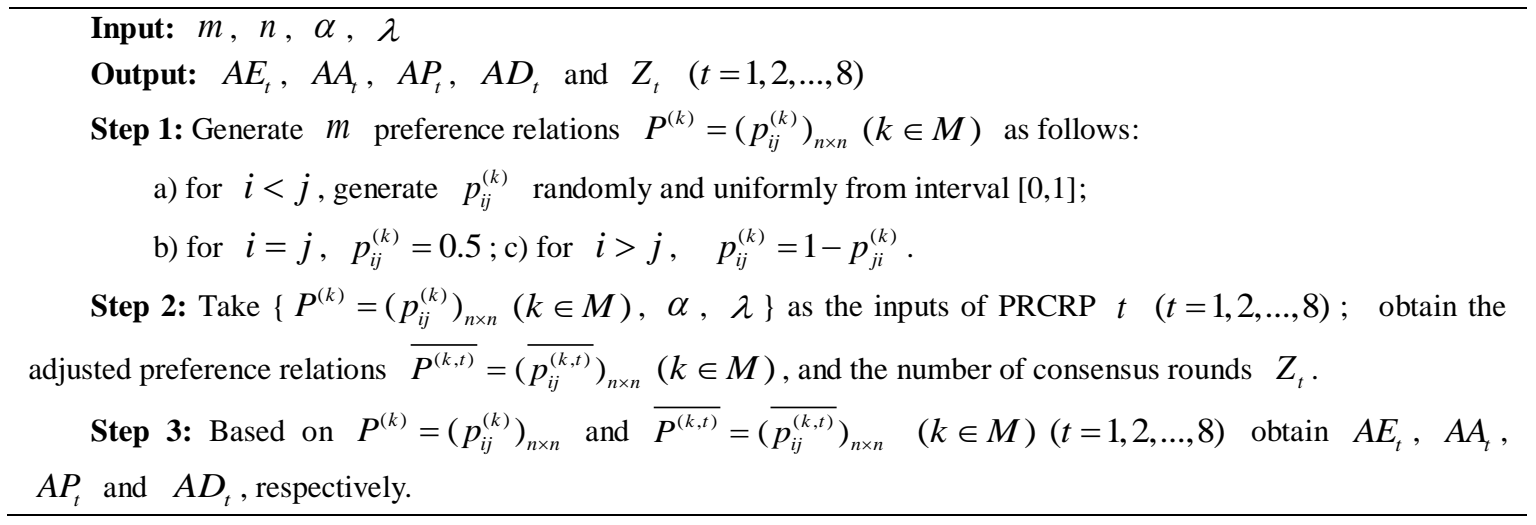

\subsection{Comparison results}

In this section, simulation results are obtained for different parameter value settings for both Simulation methods I and II, respectively.

(1) Simulation results regarding MACRPs. Setting different weight vectors of decision-makers or using different parameters combinations regarding $m, n$ and $l$ will not influence the findings of the simulation method. Additionally, we need to emphasize that when the values of $m, n$ and $l$ are large enough, similar findings can be obtained. For convenience, the decision-makers' weights are assumed in the following three combination scenarios of parameters $m, n$ and $l:$ (1) $m=3, n=5, l=3$ and $\lambda=(1 / 3,1 / 3,1 / 3)^{T} ;(2) \quad m=4, n=4, l=4$ and $\lambda=(0.25,0.25,0.25,0.25)^{T}$; (3) $m=5, n=3$, $l=4$ and $\lambda=(0.2,0.2,0.2,0.2,0.2)^{T}$. For different input parameter values of the desired consensus degree to achieve $\alpha$, Simulation method I is run 1000 times for each one of the above combination of parameters scenarios to generate average values of $A E_{t}, A A_{t}, A P_{t}, A D_{t}$ and $Z_{t}(t=1,2, \ldots, 8)$, which are shown in Figs. 1-3, respectively.

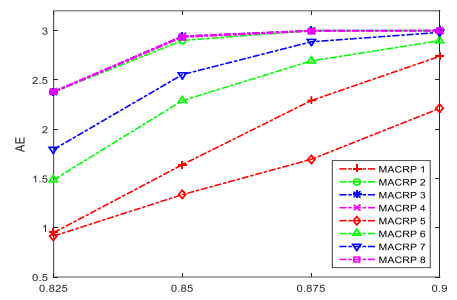

(a) $A E$

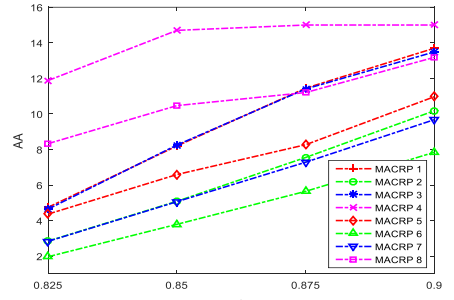

(b) $A A$

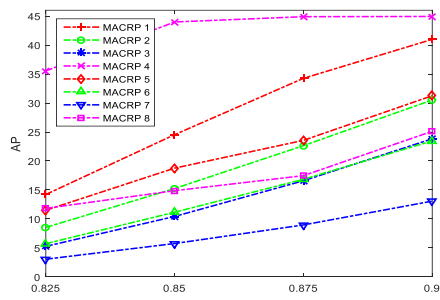

(c) $A P$

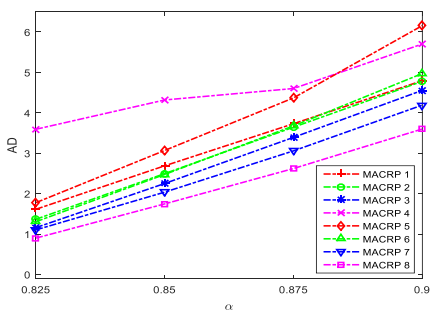

(d) $A D$

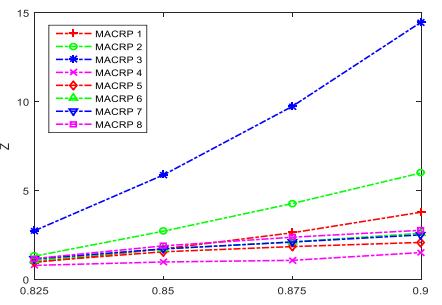

(e) $Z$

Fig. 1. The results of Simulation method I for $m=3, n=5, \quad l=3$ and $\lambda=(1 / 3,1 / 3,1 / 3)^{T}$
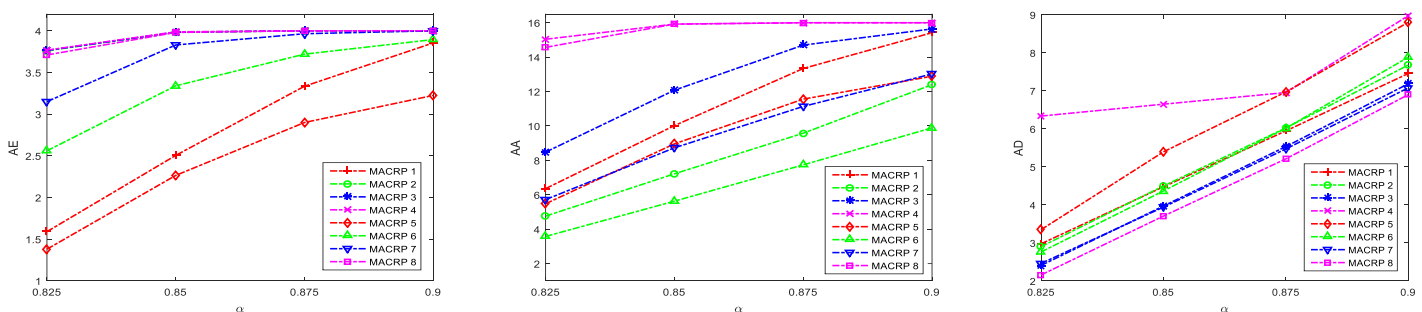

(a) $A E$
(b) $A A$
(c) $A P$

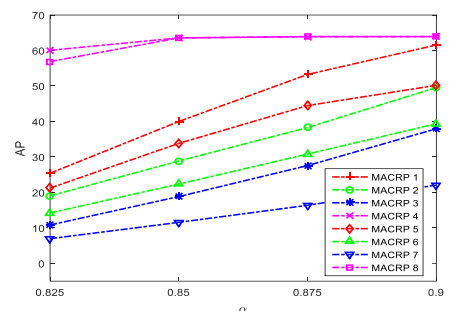

(d) $A D$

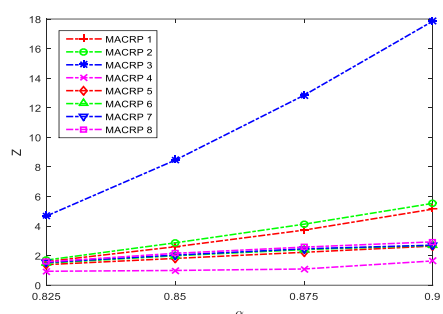

(e) $Z$

Fig. 2. The results of Simulation method I for $m=4, n=4, \quad l=4$ and $\lambda=(0.25,0.25,0.25,0.25)^{T}$

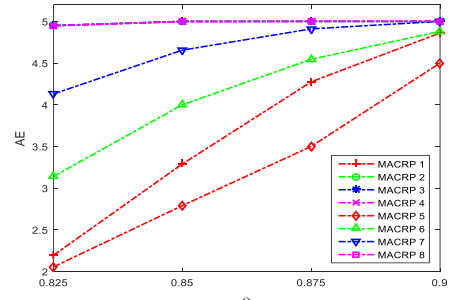

(a) $A E$

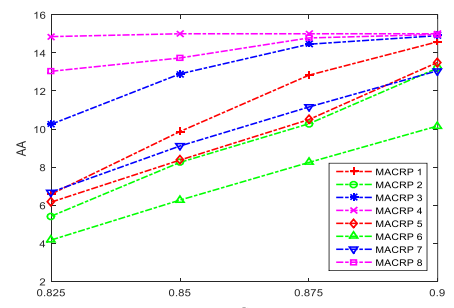

(b) $A A$

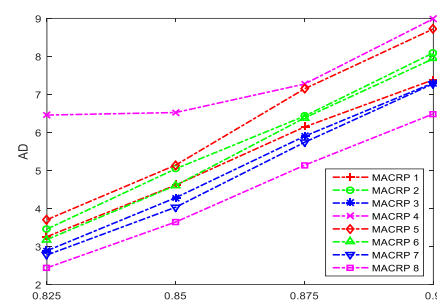

(c) $A P$

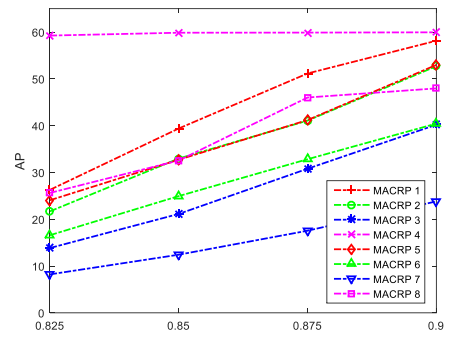

(d) $A D$

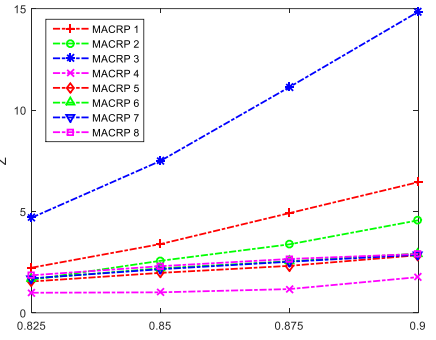

(e) $Z$

Fig. 3. The results of Simulation method I for $m=5, n=3, l=4$ and $\lambda=(0.2,0.2,0.2,0.2,0.2)^{T}$

\section{(2) Simulation results regarding PRCRPs}

In Simulation method II, the following three combination of parameters scenarios are considered: (1) $m=3, \quad n=4$ and $\lambda=(1 / 3,1 / 3,1 / 3)^{T} ;$ (2) $m=4, n=4$ and $\lambda=(1 / 4,1 / 4,1 / 4,1 / 4)^{T} ;(3)$ $m=5, \quad n=5$ and $\lambda=(1 / 5,1 / 5,1 / 5,1 / 5,1 / 5)^{T}$. Average values of $A E_{t}, A A_{t}, A P_{t}, A D_{t}$ and $Z_{t}$ for different input parameter $\alpha$ after 1000 runs under cases (1), (2) and (3) are described in Figs. 4-6, respectively.

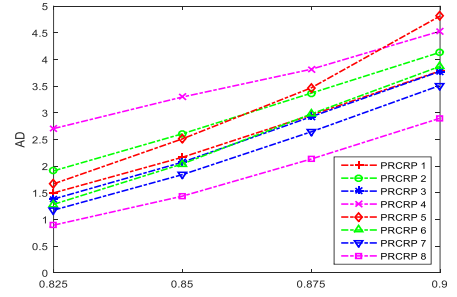

(a) $A E$

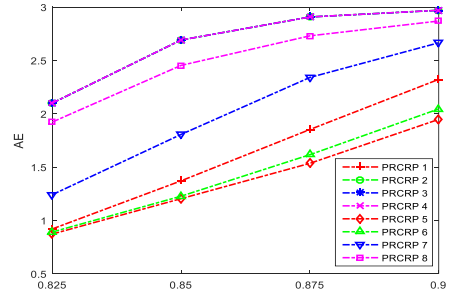

(b) $A A$

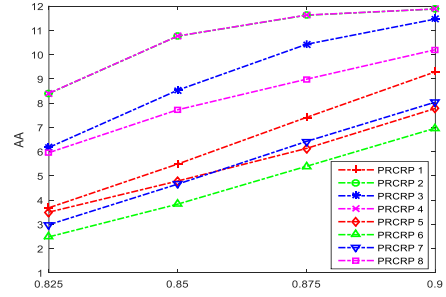

(c) $A P$

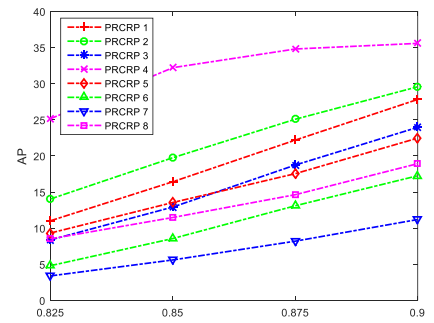

(d) $A D$

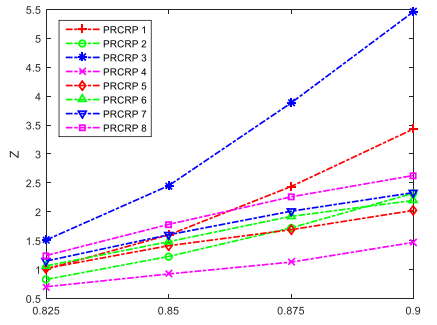

(e) $Z$ 
Fig. 4. The results of Simulation method II for $m=3, n=4$ and $\lambda=(1 / 3,1 / 3,1 / 3)^{T}$

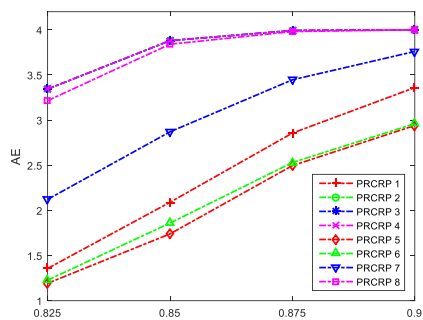

(a) $A E$

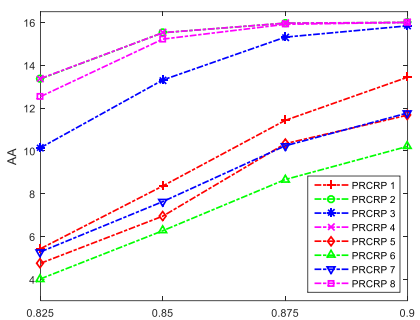

(b) $A A$

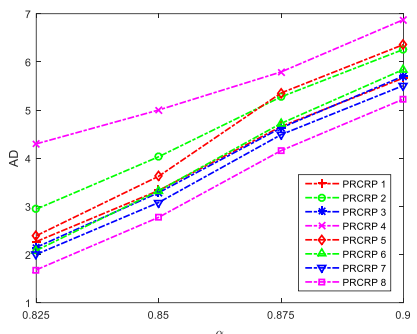

(c) $A P$

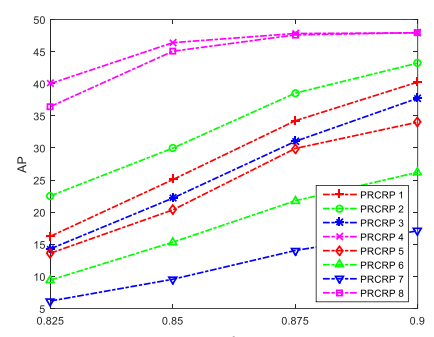

(d) $A D$

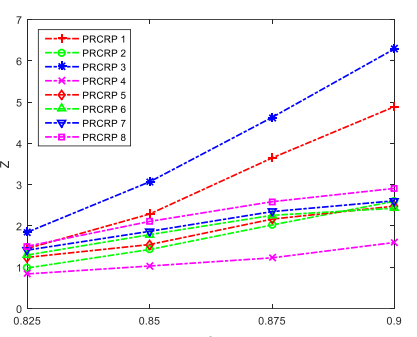

(e) $Z$

Fig. 5. The results of Simulation method II for $m=4, n=4$ and $\lambda=(1 / 4,1 / 4,1 / 4,1 / 4)^{T}$

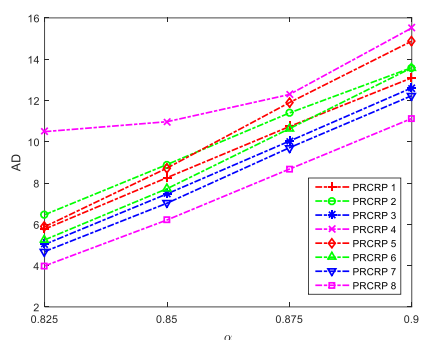

(a) $A E$

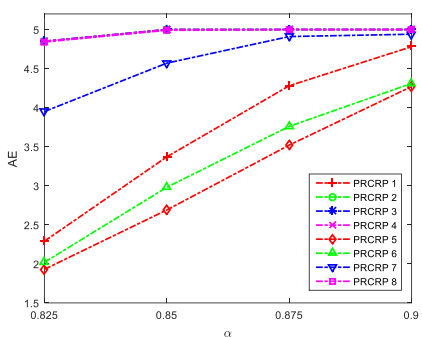

(b) $A A$

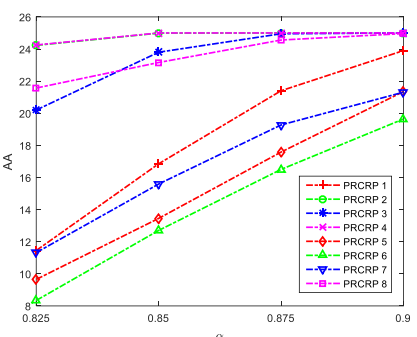

(c) $A P$

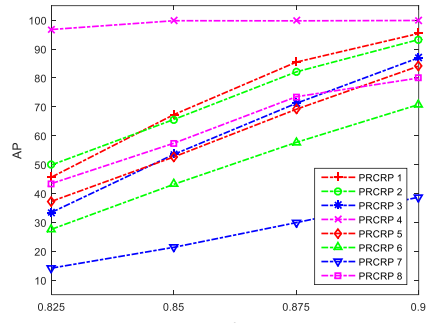

(d) $A D$

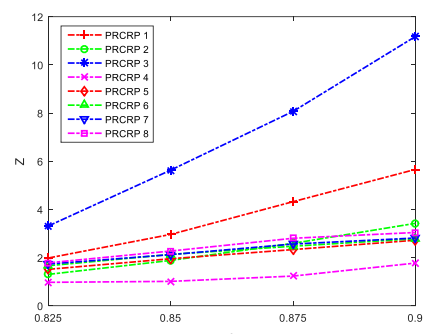

(e) $Z$

Fig. 6. The results of Simulation method II for $m=5, n=5$ and $\lambda=(1 / 5,1 / 5,1 / 5,1 / 5,1 / 5)^{T}$

From Figs. 1-6, the following observations are drawn:

(i) Average values for $A E, A A, A P, A D$ and $Z$ increase as $\alpha$ increases in all MACRPs (MACRPs 1-8) and PRCRPs (PRCRPs 1-8). This implies that adopting stricter consensus criteria in the CRP will result in higher adjustments and will lead to longer consensus processes.

(ii) With respect to the criterion $A E$, the CRPs based on the OE consensus rule (MACRP 5 and PRCRP 5), the CRPs based on the IR.E-DR consensus rule (MACRP 1 and PRCRP 1), and the CRPs based on OA consensus rule (MACRP 6 and PRCRP 6) are more efficient than the other CRPs in both MAGDM and PRGDM contexts. In particular, the CRPs based on the OE consensus rule are the most efficient with respect to the criterion $A E$.

(iii) With respect to the criterion $A A$, the CRPs based on the OA consensus rule (i.e., MACRP 6 and 
PRCRP 6) are the most efficient while the CRPs based on the DR consensus rule (i.e., MACRP 4 and PRCRP 4) are the least efficient.

(iv) With respect to the criterion $A P$, the CRPs based on the OP consensus rule (MACRP 7 and PRCRP 7) are the most efficient while the CRPs based on the DR consensus rule (MACRP 4 and PRCRP 4) are the least efficient. In addition, the efficiency of CRPs based on the OA consensus rule (MACRP 6 and PRCRP 6) is slightly worse than the efficiency of the CRPs based on the OP consensus rule.

(v) With respect to the criterion $A D$, the CRPs based on the OD consensus rule (MACRP 8 and PRCRP 8), the CPRs based on the OP consensus rule (MACRP 7 and PRCRP 7) and the CRPs based on the IR.P-DR consensus rule (MACRP 3 and PRCRP 3) are more efficient than the other CRPs in both MAGDM and PRGDM settings. In particular, the CRPs based on the OD consensus rule (MACRP 8 and PRCRP 8 ) are best while the CRPs based on the DR consensus rule (MACRP 4 and PRCRP 4) are the worst for this criterion.

(vi) With respect to the criterion $Z$, the CRPs based on the DR consensus rule (MACRP 4 and PRCRP 4) are the best while the CRPs based on the IR.P-DR consensus rule (MACRP 3 and PRCRP 3 ) are the worst. The other CRPs perform similarly with respect to this criterion.

Based on the simulation results, there is not a single CRP with best efficiency for all comparison criteria, although the CRPs based on optimization-based consensus rules were more efficient.

Note 2: (1) In the comparison analysis, we set different $\alpha$ values selected from the interval [0.8, 1], and find that the simulation results are robust under different $\alpha$ values. (2) When comparing the efficiency of PRCRPs, we only present the comparison methods and results for additive preference relations. The additive, multiplicative and linguistic preference relations are all widely used in the GDM, with the main difference among them being their corresponding domain of evaluation of the intensity of the preference. Transformation functions between additive, multiplicative and linguistic preference relations are reviewed in Chen et al. (2015). Although the specific procedures are not the same for additive, multiplicative and linguistic preference relations, they are similar. In the simulation experiments we obtain similar comparison methods and results in the cases of multiplicative and linguistic preference relations, which are not included in the paper due to space limitations. (3) It is assumed that a preference change has associated a cost in the CRPs, and a large weight of a decision-maker indicates a large unit cost to change his/her preferences. In the simulation experiments, we assumed that the weights of decision-makers are equal. Notably, we can also get the similar simulation experiments results when setting unequal decision-maker weights, which are not included due to space limitation.

\section{Multi-stage optimization-based CRPs}

In this section, we design multi-stage optimization-based CRPs under the MAGDM and PRGDM settings, respectively.

\subsection{Model design}

Simulation experiments in Section 3 illustrate that the consensus rules used to guide the feedback process have an important impact on the efficiency of CRPs. For the proposed criteria but the consensus 
rounds, the CRPs based on the optimization-based consensus rules have high efficiency. Concretely, for criteria $A E, A A, A P$ and $A D$, the most efficient CRPs are the CRPs based on the optimization-based consensus rules $O E, O A, O P$, and $O D$, respectively. In order to improve efficiency, we propose a novel consensus rule that we will call the multi-stage optimization-based consensus rule and develop a multi-stage optimization-based CRP illustrated in Fig. 7. In the proposed multi-stage optimization-based CRP, the aggregation function is used to aggregate individual preferences into a collective preference. Then, the consensus degree among the group of decision-makers is measured, and if this is below the predefined consensus degree, the adjustment suggestions are generated using multi-stage optimization-based consensus rule for supporting the preferences-modifying in the feedback process. This procedure is repeated until the predefined consensus degree among decision-makers is achieved.

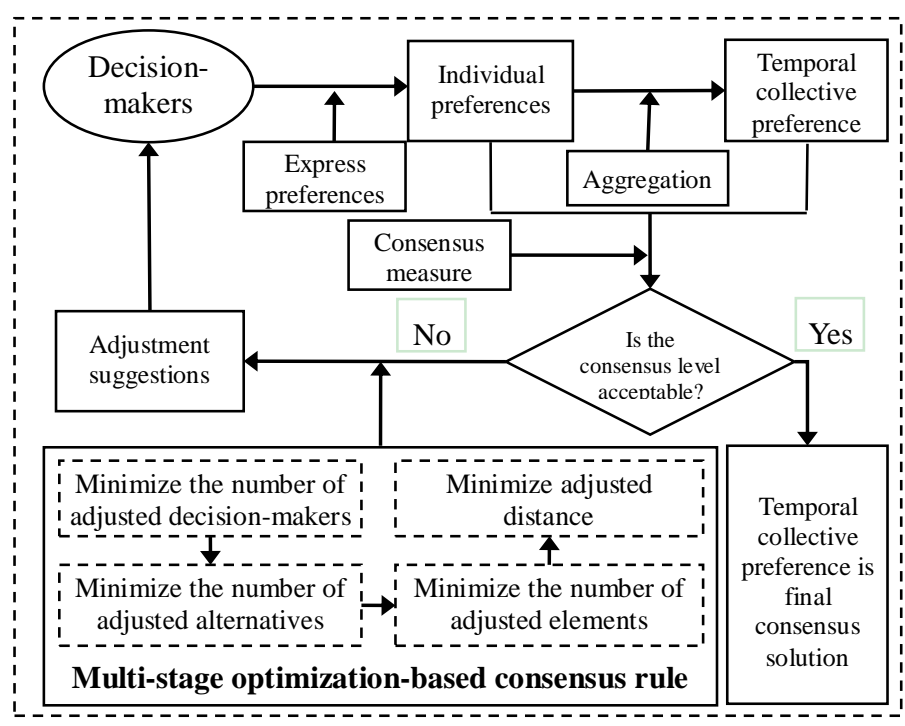

Fig. 7. The framework of the multi-stage optimization-based CRP

The multi-stage optimization-based consensus rule is applied in the following sequence: (1) the $\mathrm{OE}$ consensus rule is first used to identify the decision-makers that need to modify their preferences; (2) the OA consensus rule is then implemented to identify the alternatives whose evaluation information need to be modified; (3) following this, the OP consensus rule is utilized to identify the preference values that need change; (4) the OD consensus rule is finally employed to find the optimal adjusted individual preferences by minimizing the distance between the original and adjusted preferences.

In the following, we present the multi-stage optimization-based MACRP. For convenience, the multi-stage optimization-based MACRP is denoted as MACRP 9. The process to generate adjustment suggestions in MACRP 9 is described in Fig. 8. Using this process, we obtain the optimal adjusted decision matrices $V^{(k, *)}=\left(v_{i j}^{(k, *)}\right)_{n \times l} \quad(k \in M)$ to be presented to the decision-maker $e_{k}$ to reconsider constructing a new decision matrix $\overline{V^{(k)}}=\left(\overline{v_{i j}^{(k)}}\right)_{n \times l}$ with $\overline{v_{i j}^{(k)}} \in\left[\min \left(v_{i j}^{(k)}, v_{i j}^{(k, *)}\right), \max \left(v_{i j}^{(k)}, v_{i j}^{(c, *)}\right)\right]$.

Algorithm M-9 for MACRP 9 is Algorithm M-1 with Step 3 replaced with Step 3-H below:

Step 3-H: Adjustment generation: (1) Use model (8) to obtain $z^{(k, *)}(k \in M)$. If $z^{(k, *)}=0$, set $\overline{V_{z}^{(k)}}=V_{z}^{(k)}$ in model (11), and obtain $y_{i}^{(k, *)} \quad(k \in M ; i \in N)$. (2) If $y_{i}^{(k, *)}=0$, set $\overline{v_{i j, z}^{(k)}}=v_{i j, z}^{(k)} \quad \forall j \in L$ in model (14), and obtain $x_{i j}^{(k)} \quad(k \in M ; i \in N ; j \in L)$. (3) If $x_{i j}^{(k)}=0$, set $\overline{v_{i j, z}^{(k)}}=v_{i j, z}^{(k)}$ in model (16), and obtain $V_{z}^{(k, *)}=\left(v_{i j, z}^{(k, *)}\right)_{n \times l}(k \in M ; i \in N ; j \in L)$. When decision-maker $e_{k}$ provides $V_{z+1}^{(k)}=\left(v_{i j, z+1}^{(k)}\right)_{n \times l}$ recommend: 
$v_{i j, z+1}^{(k)} \in\left[\min \left(v_{i j, z}^{(k)}, v_{i j, z}^{(k, *)}\right), \max \left(v_{i j, z}^{(k)}, v_{i j, z}^{(k, *)}\right)\right]$.

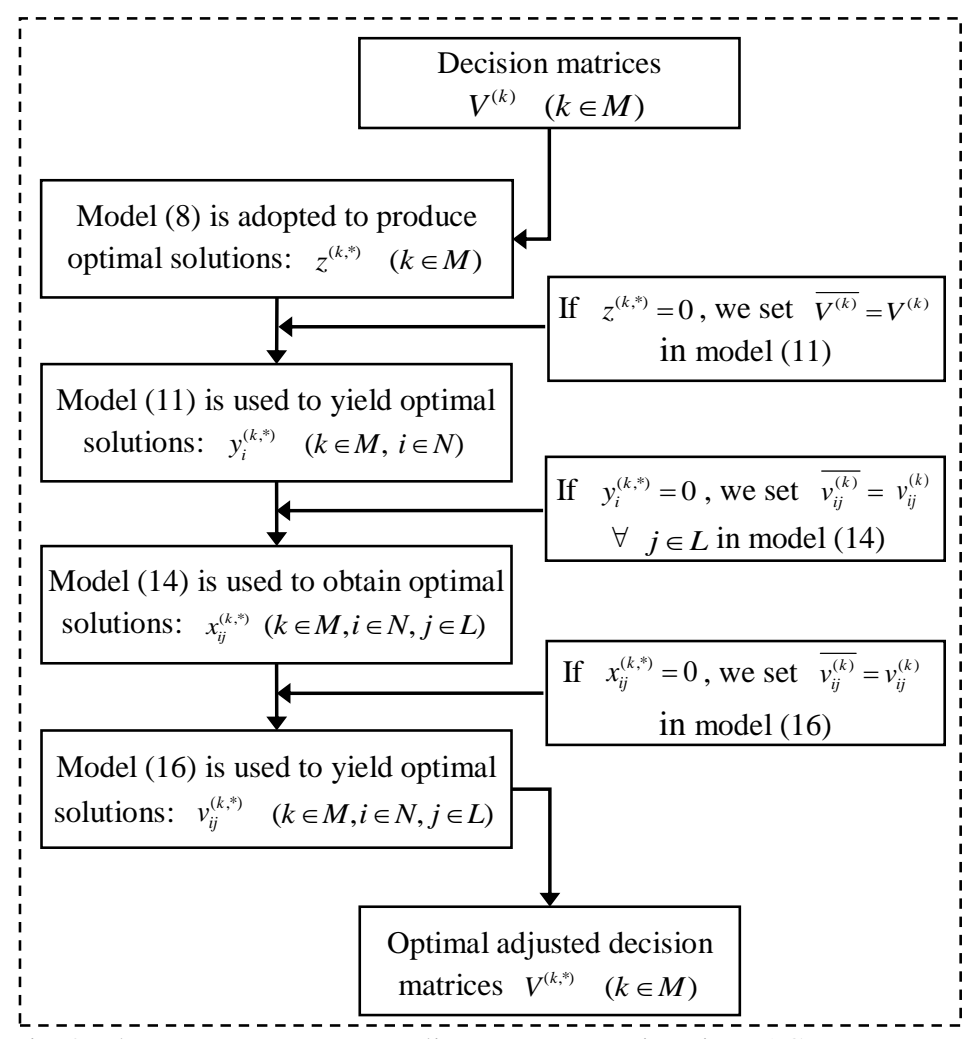

Fig. 8. The process to generate adjustment suggestions in MACRP 9

Similar to MACRP 9, a multi-stage optimization-based PRCRP, which is denoted as PRCRP 9, is developed but its detailed description is omitted due to space limitation.

\subsection{Comprehensive efficiency analysis}

In this section, we design simulation experiments to analyze the comprehensive efficiency of MACRPs and PRCRPs, respectively. MACRP 9 is added to the previous Simulation method I as one comparison object, and a new Simulation method I' is obtained. The following two sets of parameters for Simulation method I' are used: (1) $m=3, n=4, l=4$, and $\alpha=\{0.84,0.88\}$; (2) $m=5, n=3, l=3$, and $\alpha=\{0.84,0.88\} ; 1000$ runs are carried out to obtain average values of $A E, A A, A P, A D$ and $Z$ for the different MACRP as listed in Tables 6 and 7, respectively.

Table 6: The results of Simulation method I' when setting $m=3, n=4$ and $l=4$

\begin{tabular}{|c|c|c|c|c|c|c|c|c|c|c|}
\hline & \multirow{2}{*}{\multicolumn{2}{|c|}{$\frac{\mathrm{AE}}{\alpha}$}} & \multicolumn{2}{|c|}{ AA } & \multicolumn{2}{|c|}{$\mathrm{AP}$} & \multicolumn{2}{|c|}{$\mathrm{AD}$} & \multicolumn{2}{|c|}{$\mathrm{Z}$} \\
\hline & & & & & 0 & & & & 0 & \\
\hline & 0.84 & 0.88 & 0.84 & 0.88 & 0.84 & 0.88 & 0.84 & 0.88 & 0.84 & 0.88 \\
\hline MACRP 1 & 1.413 & 2.457 & 5.652 & 9.828 & 22.57 & 39.23 & 2.499 & 4.223 & 1.473 & 2.873 \\
\hline MACRP 2 & 2.853 & 3 & 3.819 & 6.858 & 15.26 & 27.41 & 2.325 & 4.195 & 1.869 & 3.852 \\
\hline MACRP 3 & 2.853 & 3 & 6.76 & 10.46 & 8.919 & 19.29 & 1.951 & 3.875 & 4.906 & 11.36 \\
\hline MACRP 4 & 2.853 & 3 & 11.41 & 12 & 45.55 & 47.87 & 4.516 & 5.019 & 0.951 & 1.112 \\
\hline MACRP 5 & 1.18 & 1.899 & 4.655 & 7.497 & 17.09 & 27.94 & 2.738 & 5.1 & 1.371 & 1.897 \\
\hline MACRP 6 & 1.952 & 2.75 & 2.817 & 5.419 & 10.98 & 21.46 & 2.239 & 4.331 & 1.509 & 2.263 \\
\hline MACRP 7 & 2.428 & 2.922 & 4.318 & 7.457 & 5.099 & 10.41 & 1.799 & 3.511 & 1.532 & 2.222 \\
\hline
\end{tabular}




\begin{tabular}{|c|c|c|c|c|c|c|c|c|c|c|}
\hline MACRP 8 & 2.843 & 2.998 & 9.127 & 10.16 & 15.24 & 20.12 & 1.538 & 2.996 & 1.687 & 2.445 \\
\hline MACRP 9 & 2.022 & 2.382 & 4.476 & 7.165 & 5.839 & 12.09 & 1.923 & 4.023 & 1.62 & 2.363 \\
\hline
\end{tabular}

Table 7: The results of Simulation method I' when setting $m=5, n=3$ and $l=3$

\begin{tabular}{|c|c|c|c|c|c|c|c|c|c|c|}
\hline & \multirow{2}{*}{\multicolumn{2}{|c|}{$\frac{\mathrm{AE}}{\alpha}$}} & \multicolumn{2}{|c|}{ AA } & \multicolumn{2}{|c|}{ AP } & \multicolumn{2}{|c|}{$\mathrm{AD}$} & \multicolumn{2}{|c|}{ Z } \\
\hline & $c$ & & & & $c$ & & $c$ & & $c$ & \\
\hline & 0.84 & 0.88 & 0.84 & 0.88 & 0.84 & 0.88 & 0.84 & 0.88 & 0.84 & 0.88 \\
\hline MACRP 1 & 2.206 & 3.672 & 11.03 & 18.36 & 44.01 & 73.29 & 4.935 & 8.094 & 2.226 & 4.126 \\
\hline MACRP 2 & 4 & 4 & 7.272 & 12.56 & 29.06 & 50.21 & 4.716 & 8.071 & 2.926 & 5.484 \\
\hline MACRP 3 & 4 & 4 & 13.74 & 18.57 & 19.18 & 37.54 & 4.2 & 7.545 & 8.696 & 17.51 \\
\hline MACRP 4 & 4 & 4 & 20 & 20 & 79.81 & 79.81 & 8.357 & 9.16 & 1 & 1.162 \\
\hline MACRP 5 & 1.896 & 2.982 & 9.318 & 14.86 & 35.22 & 57.37 & 5.688 & 9.419 & 1.62 & 2.352 \\
\hline MACRP 6 & 3.304 & 3.908 & 5.825 & 10.37 & 23.3 & 41.32 & 4.685 & 8.264 & 1.854 & 2.526 \\
\hline MACRP 7 & 3.834 & 3.994 & 9.662 & 14.51 & 12.31 & 22.23 & 4.23 & 7.427 & 1.942 & 2.52 \\
\hline MACRP 8 & 3.998 & 4 & 19.95 & 20 & 79.49 & 79.91 & 3.938 & 7.197 & 2.004 & 2.728 \\
\hline MACRP 9 & 3.146 & 3.628 & 9.424 & 13.52 & 13.1 & 24.28 & 4.311 & 7.992 & 1.92 & 2.614 \\
\hline
\end{tabular}

PRCRP 9 is also added to the Simulation method II as a comparison object and a new Simulation method II' is obtained. The following two sets of parameters for Simulation method II' are used: (1) $m=4$, $n=4$, and $\alpha=\{0.84,0.88\} ;(2) m=5, n=5$, and $\alpha=\{0.84,0.88\} ; 1000$ runs are carried out to obtain the average values of $A E, A A, A P, A D$ and $Z$ for the different PRCRPs as listed in Tables 8 and 9 , respectively.

Table 8: The results of Simulation method II' when setting $m=4$ and $n=4$

\begin{tabular}{|c|c|c|c|c|c|c|c|c|c|c|}
\hline & \multirow{2}{*}{\multicolumn{2}{|c|}{$\frac{\mathrm{AE}}{\alpha}$}} & \multirow{2}{*}{\multicolumn{2}{|c|}{$\frac{\mathrm{AA}}{\alpha}$}} & \multirow{2}{*}{\multicolumn{2}{|c|}{$\frac{\mathrm{AP}}{\alpha}$}} & \multirow{2}{*}{\multicolumn{2}{|c|}{$\frac{\mathrm{AD}}{\alpha}$}} & \multirow{2}{*}{\multicolumn{2}{|c|}{$\frac{\mathrm{Z}}{\alpha}$}} \\
\hline & & & & & & & & & & \\
\hline & 0.84 & 0.88 & 0.84 & 0.88 & 0.84 & 0.88 & 0.84 & 0.88 & 0.84 & 0.88 \\
\hline PRCRP 1 & 1.89 & 2.97 & 7.56 & 11.88 & 22.64 & 35.6 & 3.12 & 4.802 & 1.911 & 3.41 \\
\hline PRCRP 2 & 3.744 & 4 & 14.97 & 15.99 & 27.81 & 39.08 & 3.642 & 5.33 & 1.269 & 2.06 \\
\hline PRCRP 3 & 3.744 & 4 & 12.53 & 15.23 & 19.67 & 31.84 & 2.924 & 4.729 & 2.629 & 4.94 \\
\hline PRCRP 4 & 3.744 & 4 & 14.98 & 16 & 44.82 & 47.84 & 4.806 & 5.93 & 0.943 & 1.29 \\
\hline PRCRP 5 & 1.607 & 2.4 & 6.39 & 10.47 & 18.46 & 30.64 & 3.278 & 5.544 & 1.564 & 2.43 \\
\hline PRCRP 6 & 1.641 & 2.65 & 5.474 & 8.88 & 13.26 & 22.84 & 2.879 & 4.876 & 1.634 & 2.26 \\
\hline PRCRP 7 & 2.665 & 3.59 & 6.952 & 10.43 & 8.422 & 14.36 & 2.758 & 4.542 & 1.724 & 2.27 \\
\hline PRCRP 8 & 3.675 & 3.99 & 14.48 & 15.92 & 42.63 & 47.54 & 2.424 & 4.269 & 1.914 & 2.53 \\
\hline PRCRP 9 & 1.782 & 2.54 & 6.317 & 9.815 & 13.06 & 20.55 & 2.729 & 4.648 & 1.883 & 2.456 \\
\hline
\end{tabular}

Table 9: The results of Simulation method II' when setting $m=5$ and $n=5$

\begin{tabular}{|c|c|c|c|c|c|c|c|c|c|c|}
\hline & \multirow{2}{*}{\multicolumn{2}{|c|}{$\frac{\mathrm{AE}}{\alpha}$}} & \multirow{2}{*}{\multicolumn{2}{|c|}{$\frac{\mathrm{AA}}{\alpha}$}} & \multirow{2}{*}{\multicolumn{2}{|c|}{$\frac{\mathrm{AP}}{\alpha}$}} & \multirow{2}{*}{\multicolumn{2}{|c|}{$\frac{\mathrm{AD}}{\alpha}$}} & \multirow{2}{*}{\multicolumn{2}{|c|}{$\frac{\mathrm{Z}}{\alpha}$}} \\
\hline & & & & & & & & & & \\
\hline & 0.84 & 0.88 & 0.84 & 0.88 & 0.84 & 0.88 & 0.84 & 0.88 & 0.84 & 0.88 \\
\hline PRCRP 1 & 3.05 & 4.35 & 15.25 & 21.75 & 60.92 & 86.74 & 7.522 & 10.95 & 2.7 & 4.59 \\
\hline PRCRP 2 & 4.95 & 5 & 24.75 & 25 & 59.98 & 85.36 & 7.927 & 11.77 & 1.65 & 2.81 \\
\hline PRCRP 3 & 4.95 & 5 & 22.34 & 24.8 & 45.86 & 76.14 & 6.537 & 10.43 & 4.66 & 8.5 \\
\hline PRCRP 4 & 4.95 & 5 & 24.75 & 25 & 98.88 & 99.96 & 10.875 & 12.62 & 0.99 & 1.28 \\
\hline PRCRP 5 & 2.6 & 3.66 & 13 & 18.3 & 50.74 & 71.88 & 7.704 & 12.51 & 1.87 & 2.36 \\
\hline PRCRP 6 & 2.67 & 3.86 & 11.07 & 16.88 & 36.82 & 59.92 & 6.919 & 11.05 & 1.99 & 2.61 \\
\hline PRCRP 7 & 4.31 & 4.87 & 14.05 & 19.21 & 18.86 & 31.78 & 6.093 & 10.06 & 2.01 & 2.52 \\
\hline
\end{tabular}




\begin{tabular}{lllllllllll} 
PRCRP 8 & 4.91 & 4.99 & 22.32 & 24.74 & 51.14 & 77.18 & 5.366 & 9.066 & 2.13 & 2.75 \\
PRCRP 9 & 2.65 & 3.75 & 12.52 & 17.95 & 30.08 & 50.25 & 6.651 & 10.25 & 2.14 & 2.58 \\
\hline
\end{tabular}

In the following, we evaluate the comprehensive efficiency of the different MACRPs and PRCRPs. For simplicity, let $U_{i}$ denote the CRP $i$ in MAGDM or PRGDM settings, and $C A_{1}=A E, C A_{2}=A A, C A_{3}=A P$, $C A_{4}=A D$, and $C A_{5}=Z$. Let $R=\left(r_{i j}\right)_{s \times t}$ be an efficiency matrix, where $r_{i j}$ denotes the efficiency value of $U_{i}$ with respect to comparison criterion $C A_{j}$. Here, we transform $R=\left(r_{i j}\right)_{s \times t}$ into a normalized efficiency matrix $\bar{R}=\left(\overline{r_{i j}}\right)_{s \times t}$, where

$$
\overline{r_{i j}}=\frac{r_{i j}}{\sum_{i=1}^{s} r_{i j}}
$$

Next, we compute the comprehensive efficiency of the different MACRPs and PRCRPs. Let $C C E_{i}$ be the comprehensive efficiency of $U_{i}$ which is calculated as:

$$
C C E_{i}=\frac{1}{t} \sum_{j=1}^{t} \overline{r_{i j}}
$$

Clearly, the smaller the value of $C C E_{i}$, the better the comprehensive efficiency of $U_{i}$ is.

Note 3: The simulation experiments presented in Section 3 show that there is no CRP with optimal efficiency in all comparison criteria in both the MAGDM and PRGDM settings. So, we design multi-stage optimization-based CRPs and compared their comprehensive efficiency with the other types of CRPs previously analyzed. In the multi-stage optimization-based CRPs, we do not distinguish the relative importance of the five criteria and assume that they are equally important when calculating the comprehensive efficiency. Notably, the multi-stage optimization-based CRPs have the CRPs with optimization consensus rules as particular cases. For example, if we consider that the weight of criterion AE is 1 and the weights of the other criteria are 0, the multi-stage optimization the CRPs becomes the CRPs with the OE consensus rule. Consequently, the CRPs with the OE consensus rule will achieve a best comprehensive efficiency.

Based on Eq. (30), the comprehensive efficiency of the different MACRPs and PRCRPs with the considered different sets of parameters are given in Tables 10 and 11, respectively.

\begin{tabular}{|c|c|c|c|c|c|c|c|c|c|c|}
\hline & & $C C E_{1}$ & $C C E_{2}$ & $C C E_{3}$ & $C C E_{4}$ & $C C E_{5}$ & $C C E_{6}$ & $C C E_{7}$ & $C C E_{8}$ & $C C E_{9}$ \\
\hline $\begin{array}{l}m=3 \\
n=4\end{array}$ & $\alpha=0.84$ & 0.1066 & 0.1069 & 0.1418 & 0.1864 & 0.0941 & 0.0834 & 0.0819 & 0.1173 & 0.0817 \\
\hline$l=4$ & $\alpha=0.88$ & 0.122 & 0.1146 & 0.1644 & 0.1325 & 0.0997 & 0.0938 & 0.0861 & 0.101 & 0.086 \\
\hline$m=4$ & $\alpha=0.84$ & 0.1017 & 0.1022 & 0.1539 & 0.1567 & 0.0895 & 0.0826 & 0.0854 & 0.1451 & 0.0828 \\
\hline$l=4$ & $\alpha=0.88$ & 0.1209 & 0.1113 & 0.1715 & 0.1164 & 0.1 & 0.09 & 0.0858 & 0.1187 & 0.0853 \\
\hline
\end{tabular}

Table 10: The comprehensive consensus efficiency of different MACRPs

Table 11: The comprehensive consensus efficiency of different PRCRPs

\begin{tabular}{ccccccccccc}
\hline & & $C C E_{1}$ & $C C E_{2}$ & $C C E_{3}$ & $C C E_{4}$ & $C C E_{5}$ & $C C E_{6}$ & $C C E_{7}$ & $C C E_{8}$ & $C C E_{9}$ \\
\hline \multirow{2}{*}{$\begin{array}{c}m=4 \\
n=4\end{array}$} & $\alpha=0.84$ & 0.1003 & 0.1323 & 0.1317 & 0.1524 & 0.0881 & 0.0795 & 0.0869 & 0.1445 & 0.0845 \\
\cline { 2 - 10 } & $\alpha=0.88$ & 0.1153 & 0.1227 & 0.138 & 0.1249 & 0.1007 & 0.0898 & 0.0915 & 0.1275 & 0.0897 \\
\hline$m=5$ & $\alpha=0.84$ & 0.1131 & 0.1262 & 0.1426 & 0.1458 & 0.0955 & 0.0862 & 0.089 & 0.116 & 0.0856 \\
$n$ & $\alpha=0.88$ & 0.1239 & 0.1198 & 0.1519 & 0.1159 & 0.1006 & 0.0951 & 0.091 & 0.1111 & 0.0908 \\
\hline
\end{tabular}

The rankings of the MACRPs and PRCRPs based on their comprehensive efficiency are illustrated in Figs. 9 and 10, respectively. Better comprehensive efficiency are obtained with the CRPs based on the OE consensus rule (MACRP 5 and PRCRP 5), the CRPs based on the OA consensus rule (MACRP 6 and 
PRCRP 6), the CRPs based on the OP consensus rule (MACRP 7 and PRCRP 7), and the multi-stage optimization-based consensus rule (MACRP 9 and PRCRP 9) than with the CRPs with other consensus rules, with them ranked in the top 4 places in all cases, in both the MAGDM and PRGDM settings. In particular, the multi-stage optimization-based CRPs have better comprehensive efficiency in both the MAGDM and PRGDM settings. In addition, the CRPs based on the IR.P-DR consensus rule (MACRP 3 and PRCRP 3 ) and the CRPs based on the DR consensus rule (MACRP 4 and PRCRP 4) are least efficient.

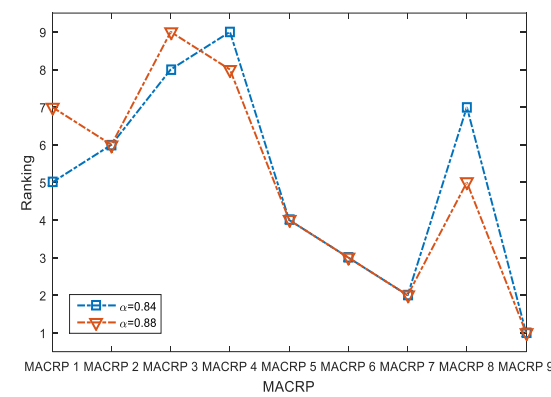

(a) $m=3, n=4, l=4$

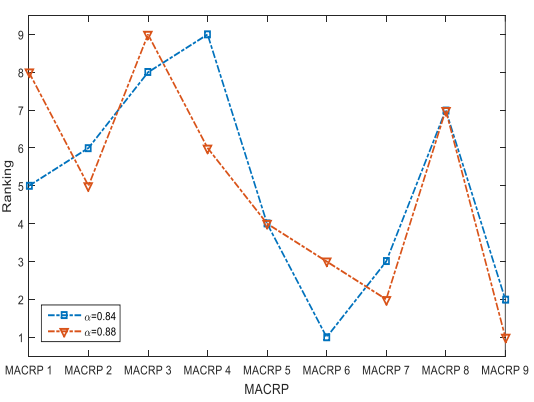

(b) $m=4, n=5, l=4$

Fig. 9. The ranking of MACRPs

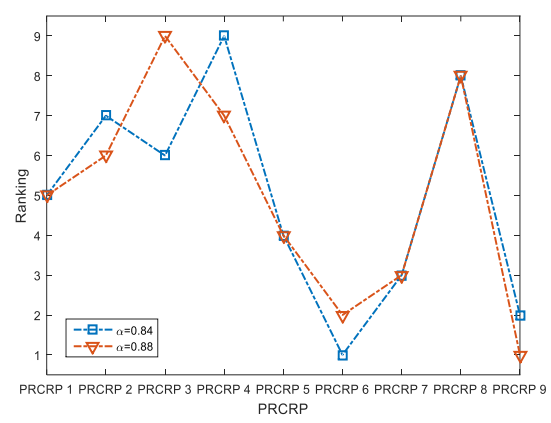

(a) $m=4, n=4$

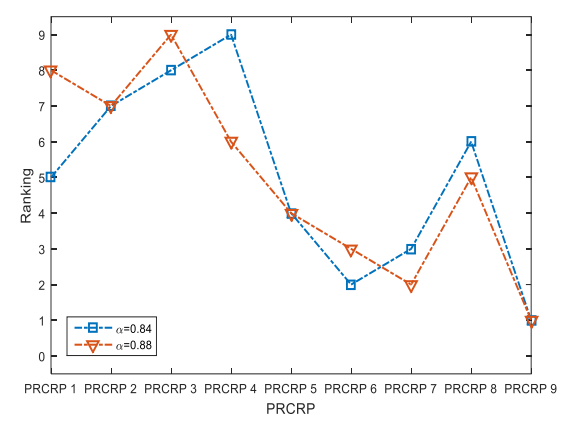

(b) $m=5, n=5$

Fig. 10. The ranking of PRCRPs

\section{Discussion: Advantages and limitations}

In this section, we point out the advantages and limitations of the present research study.

(1) Advantages: We find the following advantages of our proposal.

(i) We present a comprehensive review of existing CRPs, which are often investigated in two decision settings: MAGDM and PRGDM settings. Additionally, the study shows that CRPs are mainly based on two kinds of consensus rules: IR and DR consensus rules, and optimization-based consensus rules.

(ii) Although the minimum-adjustments is the chosen criterion by many scholars to design a CRP (e.g., Ben-Arieh et al., 2009; Ben-Arieh and Easton, 2007; Cheng et al., 2018; Dong and Xu, 2016), there is a lack of a general framework and criteria to evaluate the minimum-adjustments efficiency of the different CRPs. Following the basic idea of minimum-adjustments, this paper presents various comparison criteria for evaluating the efficiency of CRPs. Meanwhile, based on different consensus rules and decision settings, the paper presents a series of MACRPs and PRCRPs as comparison objects, which comprehensively covers the CRPs in the literature.

(iii) Detailed simulation experiments to analyze the efficiency of MACRPs and PRCRPs with respect to the presented different comparison criteria are reported and analyzed. Simulation experiments show that CRPs based on the optimization-based consensus rules are more efficient in most of the comparison criteria. However, there is no optimal CRP in all comparison criteria in both the MAGDM and PRGDM settings.

(iv) Based on the simulation analysis results regarding the existing CRPs, this paper designs multi-stage optimization-based CRPs for the MAGDM and PRGDM settings, respectively. Simulation experiment 
shows that the new multi-stage optimization-based MACRP and PRCRP have better comprehensive efficiency as measured by the normalized efficiency average of all comparison criteria.

(2) Limitations: We find the following limitations, which require of future research efforts to address them.

(i) This paper focuses on the efficiency of existing CRPs under limited resources. As mentioned in the introduction section, it is an open problem whether a CRP can improve the decision quality. Thus, we argue that it will be interesting to investigate the quality of the group decision results (such as non-biased decision results) in the proposed comparison framework based on some latest CRP studies (e.g., Gołuńska and Hołda, 2013; Kacprzyk and Zadrożny, 2016).

(ii) Because of the complexity of CRPs, simulation experiments with random data are often used to show the effectiveness of the proposed models (e.g., Palomares et al., 2014c; Quesada et al., 2015; Xu et al., 2015a), and are also adopted in this paper to evaluate the efficiency of the different CRPs analyzed. However, as shown in Regenwetter (2006), simulation experiment may result in some inaccuracy with respect to reality. So, it could be interesting in future research to investigate empirically the efficiency of CRPs.

(iii) In CRPs, some decision-makers will exhibit non-cooperative behaviors (Labella et al., 2017 and 2018; Palomares et al., 2014c; Xu et al., 2015a), which has not been considered in our simulation experiments. Therefore, it will be interesting in future research to carry out the simulation experiments with the consideration of non-cooperative behaviors. Particularly, we can use some CRP software (e.g., AFRYCA 2.0 ${ }^{1}$, see Labella et al., 2017 and 2018; Palomares et al., 2014a) to study this issue in more realistic scenarios.

\section{Conclusion}

This paper analyzed the efficiency of existing CRPs, and opened the black box of the measuring of consensus efficiency under limited resources. First, a comprehensive literature review regarding CRPs is provided, and a series of MACRPs and PRCRPs with detailed consensus reaching algorithms to implement them in practice are presented as comparison objects. Several comparison criteria under the limited resources framework are then proposed for evaluating the efficiency of the comparison objects. Following this, detailed simulation experiments are designed to compare the efficiency of MACRPs and PRCRPs with respect to the proposed comparison criteria. Further, novel multi-stage optimization-based CRPs with best comprehensive efficiency under the MAGDM and PRGDM settings, respectively, are put forward. Meanwhile, we argue that there are two interesting research avenues for future research.

(1) Modeling large-scale GDM (Liu et al., 2016; Palomares et al., 2014b; Zhang et al., 2017b) has become a trend with the development of technology and society (e.g., e-democracy (Efremov et al., 2009; Kim, 2008) and social networks (Yager, 2008)). It would be interesting to export the findings of the present research study to large-scale GDM to improve the consensus efficiency.

(2) We argue that social relationships and opinion evolution among decision-makers will be key elements in CPRs (see Dong et al., 2017 and 2018; Wu et al., 2015). In future, we plan to investigate the effects of social relationships and the opinion evolution on the decision quality and the consensus efficiency.

\section{Acknowledgments}

This work was supported by the grants (Nos. 71571124, 71871149 and 71801081) from NSF of China,

\footnotetext{
${ }^{1}$ http://sinbad2.ujaen.es/afryca/.
} 
the grants (Nos. sksyl201705 and 2018hhs-58) from Sichuan University, the grant (No. 18YJC630240) from the Chinese Ministry of Education, and the grant (No. 2017B07514) from "the Fundamental Research Funds for the Central Universities".

\section{Appendix}

Appendix A. Algorithm M-1

Table A.1: Algorithm M-1

Input: Multiple attribute decision matrices $V^{(k)}(k \in M)$, the weight vector of decision-makers $\lambda=\left(\lambda_{1}, \lambda_{2}, \ldots, \lambda_{m}\right)^{T}$, and the consensus threshold $\alpha$.

Output: The adjusted decision matrices $\overline{V^{(k)}}(k \in M)$, the adjusted group decision matrix $\overline{V^{(c)}}$, the consensus iterations $z$.

Step 1: Let $z=0$, and $V_{z}^{(k)}=\left(v_{i j, z}^{(k)}\right)_{n \times l}=\left(v_{i j}^{(k)}\right)_{n \times l}(k \in M)$.

Step 2: Use WA to obtain a group multiple attribute decision matrix $V_{z}^{(c)}=\left(v_{i j, z}^{(c)}\right)_{n \times l}$, where $v_{i j, z}^{(c)}=\sum_{k=1}^{m} \lambda_{k} \cdot v_{i j, z}^{(k)}$. Based on Eq. (5), obtain $C D_{z}\left\{e_{1}, \ldots, e_{m}\right\}$. If $C D_{z}\left\{e_{1}, \ldots, e_{m}\right\} \geq \alpha$, go to Step 4; otherwise, continue to next step.

Step 3: Let $C D_{z}\left(e_{o}\right)=\min _{k} C D_{z}\left(e_{k}\right)$. Advise decision-makers using the following procedure to provide their updated multiple attribute decision matrices $V_{z+1}^{(k)}=\left(v_{i j, z+1}^{(k)}\right)_{n \times l}$ :

$$
\left\{\begin{array}{l}
v_{i j, z+1}^{(k)} \in\left[\min \left(v_{i j, z}^{(k)}, v_{i j, z}^{(c)}\right), \max \left(v_{i j, z}^{(k)}, v_{i j, z}^{(c)}\right)\right], \quad k=o ; i \in N ; j \in L \\
v_{i j, z+1}^{(k)}=v_{i j, z}^{(k)}, \quad k \in M ; k \neq o ; i \in N ; j \in L
\end{array}\right.
$$

Let $z=z+1$. Then go to Step 2 .

Step 4: Let $\overline{V^{(k)}}=V_{z}^{(k)}(k \in M)$ and $\overline{V^{(c)}}=V_{z}^{(c)}$. Output $\overline{V^{(k)}}(k \in M), \overline{V^{(c)}}$, and $z$.

\section{Appendix B. The solving process of model (8)}

To solve model (8), two main theorems are presented.

Theorem 1: Model (8) can be equivalently transformed into the following optimization model:

$$
\begin{aligned}
& \min \sum_{k=1}^{m} z^{(k)} \\
& \text { s.t. }\left\{\begin{array}{l}
1-\frac{1}{m \times n \times l} \sum_{k=1}^{m} \sum_{i=1}^{n} \sum_{j=1}^{l}\left|\overline{v_{i j}^{(k)}}-\overline{v_{i j}^{(c)}}\right| \geq \alpha \\
\overline{v_{i j}^{(c)}}=\sum_{k=1}^{m} \lambda_{k} \cdot \overline{v_{i j}^{(k)}}, i \in N ; j \in L \\
\left(1-z^{(k)}\right) \sum_{i=1}^{n} \sum_{j=1}^{l}\left|v_{i j}^{(k)}-\overline{v_{i j}^{(k)}}\right|=0, k \in M \\
z^{(k)} \in\{0,1\}, k \in M
\end{array}\right.
\end{aligned}
$$

It is obvious, and we omit its proof.

To solve model (A.1), the following Lemma 1 is introduced.

Lemma 1 (Berthold et al., 2009): Let $x$ be a 0-1 variable. Let $\sum_{i=1}^{n} b_{i} y_{i}$ be a linear term, where $y_{i}$ $(i=1,2, \ldots, n)$ are variables with finite bounds. The product of $x$ with $\sum_{i=1}^{n} b_{i} y_{i}, x \sum_{i=1}^{n} b_{i} y_{i}$, can be replaced by a new variable $t$ and the following linear constraints:

$$
\left\{\begin{array}{l}
x y^{L} \leq t \leq x y^{U} \\
\sum_{i=1}^{n} b_{i} y_{i}-(1-x) y^{U} \leq t \leq \sum_{i=1}^{n} b_{i} y_{i}-(1-x) y^{L} \\
y^{L}=\min \sum_{i=1}^{n} b_{i} y_{i}, y^{U}=\max \sum_{i=1}^{n} b_{i} y_{i}
\end{array}\right.
$$


Theorem 2: Let $v_{i j}^{(k)}-\overline{v_{i j}^{(k)}}=c_{i j}^{(k)}, b_{i j}^{(k)}=\left|c_{i j}^{(k)}\right|, \overline{v_{i j}^{(k)}}-\overline{v_{i j}^{(c)}}=f_{i j}^{(k)}, a_{i j}^{(k)}=\left|f_{i j}^{(k)}\right|$. Then, model (A.1) can be transformed into the following model:

$$
\begin{aligned}
& \min \sum_{k=1}^{m} z^{(k)} \\
& \text { s.t. } \overline{v_{i j}^{(k)}}-\overline{v_{i j}^{(c)}}=f_{i j}^{(k)}, k \in M ; i \in N ; j \in L \\
& f_{i j}^{(k)} \leq a_{i j}^{(k)}, k \in M ; i \in N ; j \in L \\
& -f_{i j}^{(k)} \leq a_{i j}^{(k)}, k \in M ; i \in N ; j \in L \\
& \sum_{k=1}^{m} \sum_{i=1}^{n} \sum_{j=1}^{l} a_{i j}^{(k)} \leq m \times n \times l \times(1-\alpha) \\
& \overline{v_{i j}^{(k)}}-v_{i j}^{(k)}=c_{i j}^{(k)}, k \in M ; i \in N ; j \in L \\
& c_{i j}^{(k)} \leq b_{i j}^{(k)}, k \in M ; i \in N ; j \in L \\
& -c_{i j}^{(k)} \leq b_{i j}^{(k)}, k \in M ; i \in N ; j \in L \\
& \sum_{i=1}^{n} \sum_{j=1}^{l} b_{i j}^{(k)}-t^{(k)}=0, k \in M \\
& 0 \leq t^{(k)} \leq n \cdot l \cdot z^{(k)}, k \in M \\
& \sum_{i=1}^{n} \sum_{j=1}^{l} b_{i j}^{(k)}-\left(1-z^{(k)}\right) \cdot n \cdot l \leq t^{(k)} \leq \sum_{i=1}^{n} \sum_{j=1}^{l} b_{i j}^{(k)}, k \in M \\
& \sum_{k=1}^{m} \lambda_{k} \cdot \overline{v_{i j}^{(k)}}-\overline{v_{i j}^{(c)}}=0, i \in N ; j \in L \\
& z^{(k)} \in\{0,1\}, k \in M
\end{aligned}
$$

Proof: In model (A.3), (1)-(4) guarantee that $C D\left\{e_{1}, e_{2}, \ldots, e_{m}\right\}=1-\frac{1}{m \times n \times l} \sum_{k=1}^{m}\left|\overline{v_{i j}^{(k)}}-\overline{v_{i j}^{(c)}}\right| \geq \alpha$. Based on Lemma 1, (5)-(10) guarantee that $\left(1-z^{(k)}\right) \sum_{i=1}^{n} \sum_{j=1}^{l}\left|v_{i j}^{(k)}-\overline{v_{i j}^{(k)}}\right|=0$. Thus, model (A.1) can be equivalently transformed into model (A.3). This completes the proof of Theorem 2.

Theorems 1 and 2 show that the optimal solution(s) to model (8) can be equivalently obtained by solving model (A.3).

\section{Appendix C. The solving process of model (11)}

Theorems 3 and 4 prove that model (11) can be transformed into a linear programming model.

Theorem 3: Model (11) can be equivalently transformed into the following optimization model:

$$
\begin{aligned}
& \min \sum_{k=1}^{m} \sum_{i=1}^{n} y_{i}^{(k)} \\
& \text { s.t. }\left\{\begin{array}{l}
1-\frac{1}{m \times n \times l} \sum_{k=1}^{m} \sum_{i=1}^{n} \sum_{j=1}^{l}\left|\overline{v_{i j}^{(k)}}-\overline{v_{i j}^{(c)}}\right| \geq \alpha \\
\overline{v_{i j}^{(c)}}=\sum_{k=1}^{m} \lambda_{k} \cdot \overline{v_{i j}^{(k)}}, i \in N ; j \in L \\
\left(1-y_{i}^{(k)}\right) \sum_{j=1}^{l}\left|v_{i j}^{(k)}-\overline{v_{i j}^{(k)}}\right|=0, k \in M ; i \in N \\
y_{i}^{(k)} \in\{0,1\}, k \in M ; i \in N
\end{array}\right.
\end{aligned}
$$

It is obvious, and we omit its proof.

Theorem 4: Let $v_{i j}^{(k)}-\overline{v_{i j}^{(k)}}=c_{i j}^{(k)}, \quad b_{i j}^{(k)}=\left|c_{i j}^{(k)}\right|, \overline{v_{i j}^{(k)}}-\overline{v_{i j}^{(c)}}=f_{i j}^{(k)}, a_{i j}^{(k)}=\left|f_{i j}^{(k)}\right|$. Model (A.4) can be equivalently transformed into the following optimization model: 


$$
\begin{aligned}
& \min \sum_{k=1}^{m} \sum_{i=1}^{n} y_{i}^{(k)} \\
& \text { s.t. }\left\{\begin{array}{l}
\overline{v_{i j}^{(k)}}-\overline{v_{i j}^{(c)}}=f_{i j}^{(k)}, k \in M ; i \in N ; j \in L \\
f_{i j}^{(k)} \leq a_{i j}^{(k)}, k \in M ; i \in N ; j \in L \\
-f_{i j}^{(k)} \leq a_{i j}^{(k)}, k \in M ; i \in N ; j \in L \\
\sum_{k=1}^{m} \sum_{i=1}^{n} \sum_{j=1}^{l} a_{i j}^{(k)} \leq m \times n \times l \times(1-\alpha) \\
v_{i j}^{(k)}-v_{i j}^{(k)}=c_{i j}^{(k)}, k \in M ; i \in N ; j \in L \\
c_{i j}^{(k)} \leq b_{i j}^{(k)}, k \in M ; i \in N ; j \in L \\
\sum_{j=1}^{l} b_{i j}^{(k)}-t_{i}^{(k)}=0, k \in M ; i \in N ; j \in L \\
0 \leq t_{i}^{(k)} \leq l, k \in M ; i \in N \\
\sum_{j=1}^{l} b_{i j}^{(k)}-l+l y_{i}^{(k)} \leq t_{i}^{(k)} \leq \sum_{j=1}^{l} b_{i j}^{(k)}, k \in M ; i \in N \\
\overline{v_{i j}^{(c)}}=\sum_{k=1}^{m} \lambda_{k} \cdot \overline{v_{i j}^{(k)}}, i \in N ; j \in L \\
y_{i}^{(k)} \in\{0,1\}, k \in M ; i \in N
\end{array}\right.
\end{aligned}
$$

The proof process of Theorem 4 is similar to that of Theorem 2 ,

\section{Appendix D. The solving process of model (14)}

Theorems 5 and 6 show that model (14) can be transformed into a linear programming model.

Theorem 5: Model (14) can be equivalently transformed into the following optimization model:

$$
\begin{aligned}
& \min \sum_{k=1}^{m} \sum_{i=1}^{n} \sum_{j=1}^{l} x_{i j}^{(k)} \\
& \text { s.t. }\left\{\begin{array}{l}
\frac{1}{m \times n \times l} \sum_{k=1}^{m} \sum_{i=1}^{n} \sum_{j=1}^{l}\left|\overline{v_{i j}^{(k)}}-\overline{v_{i j}^{(c)}}\right| \leq 1-\alpha \\
\overline{v_{i j}^{(c)}}=W A_{\lambda}\left(\overline{v_{i j}^{(1)}}, \overline{v_{i j}^{(2)}}, \ldots, \overline{v_{i j}^{(m)}}\right), i \in N ; j \in L \\
\left(v_{i j}^{(k)}-\overline{v_{i j}^{(k)}}\right)\left(1-x_{i j}^{(k)}\right)=0, k \in M ; i \in N ; j \in L \\
x_{i j}^{(k)} \in\{0,1\}, k \in M ; i \in N ; j \in L
\end{array}\right.
\end{aligned}
$$

Theorem 6: Let $\overline{v_{i j}^{(k)}}-\overline{v_{i j}^{(c)}}=b_{i j}^{(k)}, \quad a_{i j}^{(k)}=\left|b_{i j}^{(k)}\right|, \quad t_{i j}^{(k)}=\overline{v_{i j}^{(k)}} \cdot x_{i j}^{(k)}$. Model (A.6) can be equivalently transformed into the following optimization model: 


$$
\begin{aligned}
& \min \sum_{k=1}^{m} \sum_{i=1}^{n} \sum_{j=1}^{l} x_{i j}^{(k)} \\
& \text { s.t. }\left\{\begin{array}{l}
\overline{v_{i j}^{(k)}}-\overline{v_{i j}^{(c)}}=b_{i j}^{(k)}, k \in M ; i \in N ; j \in L \\
b_{i j}^{(k)} \leq a_{i j}^{(k)}, k \in M ; i \in N ; j \in L \\
-b_{i j}^{(k)} \leq a_{i j}^{(k)}, k \in M ; i \in N ; j \in L \\
\sum_{k=1}^{m} \sum_{i=1}^{n} \sum_{j=1}^{l} a_{i j}^{(k)} \leq m \times n \times l \times(1-\alpha) \\
t_{i j}^{(k)}-x_{i j}^{(k)} \leq 0, k \in M ; i \in N ; j \in L \\
v_{i j}^{(k)}+x_{i j}^{(k)}-1-t_{i j}^{(k)} \leq 0, k \in M ; i \in N ; j \in L \\
t_{i j}^{(k)}-v_{i j}^{(k)} \leq 0, k \in M ; i \in N ; j \in L \\
v_{i j}^{(k)}-\overline{v_{i j}^{(k)}}-v_{i j}^{(k)} x_{i j}^{(k)}+t_{i j}^{(k)}=0, k \in M ; i \in N ; j \in L \\
\overline{v_{i j}^{(c)}}=\sum_{k=1}^{m} \lambda_{k} \cdot \overline{v_{i j}^{(k)}}, i \in N ; j \in L \\
x_{i j}^{(k)} \in\{0,1\}, k \in M ; i \in N ; j \in L
\end{array}\right.
\end{aligned}
$$

The proof process of Theorem 6 is similar to that of Theorem 2

\section{Appendix E. The solving process of model (16)}

Theorem 7 shows that model (16) can be transformed into a linear programming model.

Theorem 7: Let $v_{i j}^{(k)}-\overline{v_{i j}^{(k)}}=c_{i j}^{(k)}, \quad a_{i j}^{(k)}=\left|c_{i j}^{(k)}\right|, \overline{v_{i j}^{(k)}}-\overline{v_{i j}^{(c)}}=f_{i j}^{(k)}, \quad b_{i j}^{(k)}=\left|f_{i j}^{(k)}\right|$. Model (16) can be equivalently transformed into the following optimization model:

$$
\begin{aligned}
& \min \sum_{k=1}^{m} \sum_{i=1}^{n} \sum_{j=1}^{l} a_{i j}^{(k)} \\
& \text { s.t. }\left\{\begin{array}{l}
v_{i j}^{(k)}-\overline{v_{i j}^{(k)}}=c_{i j}^{(k)}, k \in M ; i \in N ; j \in L \\
c_{i j}^{(k)} \leq a_{i j}^{(k)}, k \in M ; i \in N ; j \in L \\
-c_{i j}^{(k)} \leq a_{i j}^{(k)}, k \in M ; i \in N ; j \in L \\
\overline{m \times n \times l} \sum_{k=1}^{m} \sum_{i=1}^{n} \sum_{j=1}^{l} b_{i j}^{(k)} \leq 1-\alpha \\
\overline{v_{i j}^{(k)}}-\overline{v_{i j}^{(c)}}=f_{i j}^{(k)}, k \in M ; i \in N ; j \in L \\
f_{i j}^{(k)} \leq b_{i j}^{(k)}, k \in M ; i \in N ; j \in L \\
-f_{i j}^{(k)} \leq b_{i j}^{(k)}, k \in M ; i \in N ; j \in L \\
\overline{v_{i j}^{(c)}}=\sum_{k=1}^{m} \lambda_{k} \cdot \overline{v_{i j}^{(k)}}, i \in N ; j \in L
\end{array}\right.
\end{aligned}
$$

Proof: In model (A.8), (1)-(3) guarantee that $\left|v_{i j}^{(k)}-\overline{v_{i j}^{(k)}}\right| \leq a_{i j}^{(k)}$. The objective function achieves optimum value only when $\left|v_{i j}^{(k)}-\overline{v_{i j}^{(k)}}\right|=a_{i j}^{(k)}$. In addition, (4)-(8) guarantee $C D\left\{e_{1}, e_{2}, \ldots, e_{m}\right\} \geq \alpha$. Thus, model (16) can be equivalently transformed into model (A.8). This completes the proof of Theorem 7.

\section{Reference}

Aguarón, J., Escobar, M.T., Moreno-Jiménez, J.M., (2016). The precise consistency consensus matrix in a local AHP-group decision making context. Annals of Operations Research 245, 245-259.

Alonso, S., Herrera-Viedma E., Chiclana, F., Herrera, F., (2010). A web based consensus support system for group decision making problems and incomplete preferences. Information Sciences 180, 4477-4495.

Alonso, S., Pérez, I.J., Cabrerizo, F.J., Herrera-Viedma, E., (2013). A linguistic consensus model for Web 2.0 communities. Applied Soft Computing 13, 149-157. 
Altuzarra, A., Moreno-Jiménez, J.M., Salvador, M., (2010). Consensus building in AHP-group decision making: A bayesian approach. Operations Research 58, 1755-1773.

Arrow, K.J., (1951). Individual Values and Social Choice. Wiley, New York.

Ben-Arieh, D, Chen, Z.F., (2006). Linguistic-labels aggregation and consensus measure for autocratic decision making using group recommendations. IEEE Transactions on Systems, Man and Cybernetics, Part A: Systems and Humans 36, 558-568.

Ben-Arieh, D., Easton, T., (2007). Multi-criteria group consensus under linear cost opinion elasticity. Decision Support Systems 43, 713-721.

Ben-Arieh, D., Easton, T., Evans, B., (2009). Minimum cost consensus with quadratic cost functions. IEEE Transactions on Systems, Man and Cybernetics, Part A: Systems and Humans 39, 210-217.

Berthold, T., Heinz, S., Vigerske, S., (2009). Extending a cip framework to solve MIQCPS. ZIB-Report 09-23, Zuse Institute Berlin.

Bezdek, J.C., Spillman, B., Spillman, R., (1978). A fuzzy relation space for group decision theory. Fuzzy Sets and Systems 1, 255-268.

Brunelli, M., Fedrizzi, M., Fedrizzi, M., (2014). Fuzzy m-ary adjacency relations in social network analysis: Optimization and consensus evaluation. Information Fusion 17, 36-45.

Cabrerizo, F.J., Alonso, A., Herrera-Viedma, E., (2009). A consensus model for group decision making problems with unbalanced fuzzy linguistic information. International Journal of Information Technology \& Decision making 8, 109-131.

Cabrerizo, F.J., Pérez, I.J., Herrera-Viedma, E., (2010). Managing the consensus in group decision making in an unbalanced fuzzy linguistic context with incomplete information. Knowledge-Based Systems 23, 169-181.

Cao, M., Morse, A., Anderson, B., (2008). Reaching a consensus in a dynamically changing environment: A graphical approach. SIAM Journal on Control and Optimization 47, 575-600.

Chen, X., Zhang, H.J., Dong, Y.C., (2015). The fusion process with heterogeneous preference structures in group decision making: A survey. Information Fusion 24, 72-83.

Cheng, D., Zhou, Z.L., Cheng, F.X., Zhou, Y.F., Xie, Y.J., (2018). Modeling the minimum cost consensus problem in an asymmetric costs context. European Journal of Operational Research 270, 1122-1137.

Chiclana, F., Garcia, J.M.T., Del Moral, M.J., Herrera-Viedma, E., (2013). A statistical comparative study of different similarity measures of consensus in group decision making. Information Sciences 221, 110-123.

Chiclana, F., Mata, F., Martínez, L., Herrera-Viedma, E., Alonso, S., (2008). Integration of a consistency control module within a consensus model. International Journal of Uncertainty, Fuzziness and Knowledge-Based Systems 16, 35-53.

Choudhury, A.K., Shankar, R., Tiwari, M.K., (2006). Consensus-based intelligent group decision-making model for the selection of advanced technology. Decision Support Systems 42, 1776-1799.

Coch, L., French, J.R.P., (1948). Overcoming resistance to change. Human Relations 1, 512-532.

Cook, W.D., (2006). Distance-based and ad hoc consensus models in ordinal preference ranking. European Journal of Operational Research 172 (2006) 369-385.

Cook, W.D., Kress, M., (1985). Ordinal ranking with intensity of preference. Management Science 31, 26-32.

Dong, Q.X., Cooper, O., (2016). A peer-to-peer dynamic adaptive consensus reaching model for the group AHP decision making. European Journal of Operational Research 250, 521-530.

Dong, Y.C., Chen, X., Herrera, F., (2015a). Minimizing adjusted simple terms in the consensus reaching process with hesitant linguistic assessments in group decision making. Information Sciences 297, 95-117.

Dong, Y.C., Ding, Z.G., Martínez, L., Herrera, F., (2017). Managing consensus based on leadership in opinion dynamics. Information Sciences 397-398, 187-205.

Dong, Y.C., Li, C.C., YXu, Y.F., Gu, X., (2015b). Consensus-based group decision making under multi-granular unbalanced 2-tuple linguistic preference relations. Group Decision and Negotiation 24, 217-242. 
Dong, Y.C., Xu, J.P., (2016). Consensus building in group decision making: Searching the consensus path with minimum adjustments. Springer-Verlag.

Dong, Y.C., Xu, Y.F., Li, H.Y., Feng, B., (2010a). The OWA-based consensus operator under linguistic representation models using position indexes. European Journal of Operational Research 203, 455-463.

Dong, Y.C., Zhan, M., Kou, G., Ding, Z.G., Liang, H.M., (2018). A survey on the fusion process in opinion dynamics. Information Fusion 43, 57-65.

Dong, Y.C., Zhang, G.Q., Hong, W.C., Xu, Y.F., (2010b). Consensus models for AHP group decision making under row geometric mean prioritization method. Decision Support Systems 49, 281-289.

Dong, Y.C., Zhang, H.J., (2014). Multiperson decision making with different preference representation structures: A direct consensus framework and its properties. Knowledge-Based Systems 58, 45-57.

Dong, Y.C., Zhang, H.J., Herrera-Viedma, E., (2016a). Consensus reaching model in the complex and dynamic MAGDM problem. Knowledge-Based Systems 106, 206-219.

Dong, Y.C., Zhang, H.J., Herrera-Viedma, E., (2016b). Integrating experts-weights generated dynamically into the consensus reaching process and its applications in managing non-cooperative behaviors. Decision Support Systems 84, 1-15.

Efremov, R., Insua, D.R., Lotov, A., (2009). A framework for participatory decision support using Pareto frontier visualization, goal identification and arbitration. European Journal of Operational Research 199, 459-467.

Erdamar, B., García-Lapresta, J.L., Pérez-Román, D., Remzi Sanver, M., (2014). Measuring consensus in a preference-approval context. Information Fusion 17, 14-21.

Escobar, M.T., Aguarón, J., Moreno-Jiménez, J.M., (2015). Some extensions of the precise consistency consensus matrix. Decision Support Systems 74, 67-77.

Escobar, M.T., Moreno-Jiménez, J.M., (2007). Aggregation of individual preference structures in AHP-group decision making. Group Decision and Negotiation 16, 287-301.

Fedrizzi, M., Fedrizzi, M., Pereira, R.A., (1999). Soft consensus and network dynamics in group decision making. International Journal of Intelligent Systems 14, 63-77.

Fedrizzi, M., Giove, S., (2013). Optimal sequencing in incomplete pairwise comparisons for large-dimensional problems. International Journal of General Systems 42, 366-375.

Fedrizzi, M., Kacprzyk, J., Zadrożny, S., (1988). An interactive multi-user decision support system for consensus reaching processes using fuzzy logic with linguistic quantifiers. Decision Support Systems 4, 313-327.

Fishburn, P.C., (1973). The theory of social choice. Princeton University Press.

French, J.R.P., (1956). A formal theory of social power. Psychological Review 63, 181-194.

French, S., (1981). Consensus of opinion. European Journal of Operational Research 7, 332-340.

Gołuńska, D., Hołda, M., (2013). The need of fairness in group consensus reaching process in a fuzzy environment, Czasopismo Techniczne, Automatyka 1-AC, 29-38.

Gong, Z.W., Xu, C., Chiclana, F., Xu, X.X., (2017). Consensus measure with multi-stage fluctuation utility based on China's urban demolition negotiation. Group Decision and Negotiation 26, 379-407.

Gong, Z.W., Xu, X.X., Lu, F.L., Li, L.S., Xu, C., (2015a). On consensus models with utility preferences and limited budget. Applied Soft Computing 35, 840-849.

Gong, Z.W., Xu, X.X., Zhang, H.H., Ozturk, U.A., Herrera-Viedma, E., Xu, C., (2015b). The consensus models with interval preference opinions and their economic interpretation. Omega 55, 81-90.

Gong, Z.W., Zhang, H.H., Forrest, J., Li, L., Xu, X., (2015c). Two consensus models based on the minimum cost and maximum return regarding either all individuals or one individual. European Journal of Operational Research 240, 183-192.

González-Arteaga, T., De Andrés Calle, R., Chiclana, F., (2016). A new measure of consensus with fuzzy preference relations: The correlation consensus degree. Knowledge-Based Systems 107,104-116. 
Greco, S., Kadziński, M., Mousseau, V., Słowiński, R., (2012). Robust ordinal regression for multiple criteria group decision: UTAGMS-GROUP and UTADISGMS-GROUP. Decision Support Systems 52, 549-561.

Greco, S., Mousseau, V., Słowiński, R., (2014). Robust ordinal regression for value functions handling interacting criteria. European Journal of Operational Research 239, 711-730.

Groot, M.M.D., (1974). Reaching a consensus. Journal of the American Statistical Association 69, 118-121.

Guha, D., Chakraborty, D., (2011). Fuzzy multi attribute group decision making method to achieve consensus under the consideration of degrees of confidence of experts' opinions. Computers \& Industrial Engineering 60, 493-504.

Harary, F., (1959). On the measurement of structural balance. Behavioral Science 4, 316-323.

Hartmann, S., Martini, C., Sprenger, J., (2009). Consensual Decision-Making Among Epistemic Peers. Episteme 6, 110-129.

Herrera, F., Herrera-Viedma, E., Verdegay, J.L., (1997). A rational consensus model in group decision making using linguistic assessments. Fuzzy Sets and Systems 88, 31-49.

Herrera-Viedma, E., Alonso, S., Chiclana, F., Herrera, F., (2007a). A consensus model for group decision making with incomplete fuzzy preference relations. IEEE Transactions on Fuzzy Systems 15, 863-877.

Herrera-Viedma, E., Cabrerizo, F.J., Kacprzyk, J., Pedrycz, W., (2014). A review of soft consensus models in a fuzzy environment. Information Fusion 17, 4-13.

Herrera-Viedma, E., Chiclana, F., Herrera, F., Alonso, S., (2007b). Group decision-making model with incomplete fuzzy preference relations based on additive consistency. IEEE Transactions on Systems, Man, and Cybernetics, Part B: Cybernetics 37, 176-189.

Herrera-Viedma, E., Herrera, F., Chiclana, F., (2002). A consensus model for multiperson decision making with different preference structures. IEEE Transactions on Systems, Man and Cybernetics, Part A: Systems and Humans 32, 394-402.

Herrera-Viedma, E., Martinez, L., Mata, F., Chiclana, F., (2005). A consensus support system sodel for group decision-making problems with multigranular linguistic preference relations. IEEE Transactions on Fuzzy Systems 13, 644-658.

Hochbaum, D.S., Levin, A., (2006). Methodologies and algorithms for group-rankings decision. Management Science 52, 1394-1408.

Kacprzyk, J., Fedrizzi, M., (1988). A 'soft' measure of consensus in the setting of partial (fuzzy) preferences. European Journal of Operational Research 34, 316-325.

Kacprzyk, J., Fedrizzi, M., Nurmi, H., (1992). Group decision making and consensus under fuzzy preferences and fuzzy majority. Fuzzy Sets and Systems 49, 21-31.

Kacprzyk, J., Fedrizzi, M., Nurmi, H., (1997). Soft degrees of consensus under additive preferences and fuzzy majorities, in J. Kacprzyk, H. Nurmi, M. Fedrizzi (Eds.): Consensus under Fuzziness, Kluwer, Boston, 55-81.

Kacprzyk, J., Zadrożny, S., (2010a). Soft computing and Web intelligence for supporting consensus reaching. Soft Computing $14,833-846$.

Kacprzyk, J., Zadrożny, S., (2016). On a fairness type approach to consensus reaching support under fuzziness via linguistic summaries, IEEE International Conference on Fuzzy Systems (FUZZ-IEEE), 1999-2006.

Kacprzyk, J., Zadrozny, S., Ras, Z.W., (2010b). How to support consensus reaching using action rules: a novel approach. International Journal of Uncertainty, Fuzziness and Knowledge-Based Systems 18, 451-470.

Kim, J., (2008). A model and case for supporting participatory public decision making in e-democracy. Group Decision and Negotiation 17, 179-193.

Kim, S.H., Choi, S.H., Kim, J.K., (1999). An interactive procedure for multiple attribute group decision making with incomplete information: Range-based approach. European Journal of Operational Research 118, 139-152.

Kozierkiewicz-Hetmańska, A., (2017). The analysis of expert opinions' consensus quality. Information Fusion 34, 80-86.

Lawryshyn, Y., Collan, M., Luukka, P., Fedrizzi, M., (2017). New procedure for valuing patents under imprecise information with a consensual dynamics model and a real options framework. Expert Systems with Applications 86, 155-1564.

Labella, Á., Liu, Y., Rodríguez, R.M., Martínez, L., (2018). Analyzing the performance of classical consensus models in large 
scale group decision making: A comparative study. Applied Soft Computing 67, 677-690.

Labella, Á., Martínez, L., Rodríguez, R.M., (2017). Can classical consensus models deal with large scale group decision making? 12th International Conference on Intelligent Systems and Knowledge Engineering (ISKE), 1-7.

Lee, H.S., (2002). Optimal consensus of fuzzy opinions under group decision making environment. Fuzzy Sets and Systems 132, 303-315.

Lehrer, K., Wagner, C., (1994). Rational Consensus in Science and Society. Dordrecht, Reidel.

Li, C.C., Dong, Y.C., Herrera, F., Herrera-Viedma, E., Martínez, L., (2017). Personalized individual semantics in computing with words for supporting linguistic group decision making. An application on consensus reaching. Information Fusion 33, $29-40$.

Li, C.C., Rodríguez, R.M., Martínez, L., Dong, Y.C., Herrera, F., (2018). Consensus building with individual consistency control in group decision making. IEEE Transactions on Fuzzy Systems, in press, doi: 10.1109/TFUZZ.2018.2856125.

Liu, Y., Fan, Z.P., Zhang, X., (2016). A method for large group decision-making based on evaluation information provided by participators from multiple groups. Information Fusion, 132-141.

Ma, J., Lu, J., Zhang, G., (2014). A three-level-similarity measuring method of participant opinions in multiple-criteria group decision supports. Decision Support Systems 59, 74-83.

Maier, K., Stix, V., (2013). A semi-automated approach for structuring multi criteria decision problems. European Journal of Operational Research 225, 487-496.

Martel, J.M., Kiss, L., (1994). A support to consensus reaching in group decision. Group Decision and Negotiation 3, 93-119.

Mata, F., Martinez, L., Herrera-Viedma, E., (2009). An adaptive consensus support model for group decision-making problems in a multigranular fuzzy linguistic context. IEEE Transactions on Fuzzy Systems 17, 279-290.

Meng, F.Y., An, Q.X., Chen, X.H., (2016). A consistency and consensus-based method to group decision making with interval linguistic preference relations. Journal of the Operational Research Society 67, 1-19.

Moreno-Jiménez, J.M., Aguarón, J., Raluy, A., Turón, A., (2005). A spreadsheet module for consistent AHP-consensus building. Group Decision and Negotiation 14, 89-108.

Moreno-Jiménez, J.M., Aguarón, J., Escobar, M.T., (2008). The core of consistency in AHP-group decision making. Group Decision and Negotiation 17, 249-265.

Moreno-Jiménez, J.M., Pérez-Espés, C., Wimmer, M., (2013). The effectiveness of E-Governance experiences in the knowledge society. 13th European Conference on e-Government (ECEG), 354-363.

Moreno-Jiménez, J.M., Salvador, M., Gargallo, P., Altuzarra, A., (2016). Systemic decision making: A bayesian approach in AHP. Annals of Operations Research 245, 261-284.

Orlovsky, S.A., (1978). Decision-making with a fuzzy preference relation. Fuzzy Sets and Systems 1, 155-167.

Palomares, I., Estrella, F.J., Martínez, L., Herrera, F., (2014a). Consensus under a fuzzy context: Taxonomy, analysis framework AFRYCA and experimental case of study. Information Fusion 20, 252-271.

Palomares, I., Martínez, L., Herrera, F., (2014b). MENTOR: A graphical monitoring tool of preferences evolution in large-scale group decision making. Knowledge-Based Systems 58, 66-74.

Palomares, I., Martinez, L., Herrera, F., (2014c). A consensus model to detect and manage noncooperative behaviors in large-scale group decision making. IEEE Transactions on Fuzzy Systems 22, 516-530.

Parreiras, R.O, Ekel, P.Y, Bernardes, F.G, (2012a). A dynamic consensus scheme based on a nonreciprocal fuzzy preference relation modeling. Information Sciences 211, 1-17.

Parreiras, R.O., Ekel, P.Y., Martini, J.S.C., Palhares, R.M., (2010). A flexible consensus scheme for multicriteria group decision making under linguistic assessments. Information Sciences 180, 1075-1089.

Parreiras, R.O., Ekel, P.Y., Morais, D.C., (2012b). Fuzzy set based consensus schemes for multicriteria group decision making applied to strategic planning. Group Decision and Negotiation 21, 153-183.

Pedrycz, W., Song, M., (2011). Analytic hierarchy process (AHP) in group decision making and its optimization with an 
allocation of information granularity. IEEE Transactions on Fuzzy Systems 19, 527-539.

Pérez, I.J., Cabrerizo, F.J., Alonso, S., Dong, Y.C., Chiclana, F., Herrera-Viedma, E., (2018). On dynamic consensus processes in group decision making problems. Information Sciences 459, 20-35.

Pérez, I.J., Cabrerizo, F.J., Alonso, S., Herrera-Viedma, E., (2014). A new consensus model for group decision making problems with non-homogeneous experts. IEEE Transactions on Systems, Man, and Cybernetics: Systems 44, 494-498.

Pérez, I.J., Cabrerizo, F.J., Herrera-Viedma, E., (2010). A mobile decision support system for dynamic group decision-making problems. IEEE Transactions on Systems, Man and Cybernetics, Part A: Systems and Humans 40, 1244-1256.

Quesada, F.J., Palomares, I., Martínez, L., (2015). Managing experts behavior in large-scale consensus reaching processes with uninorm aggregation operators. Applied Soft Computing 35, 873-887.

Rega, H.M., Colyvan, M., Markovchick-Nicholls, L., (2006). A formal model for consensus and negotiation in environmental management. Journal of Environmental Management 80, 167-176.

Regenwetter, M., (2006). Behavioral social choice: probabilistic models, statistical inference, and applications. Cambridge University Press.

Roberto, M.A., (2005). Why making the decisions the right way is more important than making the right decisions. Ivey Business Journal.

Saaty, T.L., (1980). The analytic hierarchy process, McGraw-Hill, New York.

Srdjevic, B., (2007). Linking analytic hierarchy process and social choice methods to support group decision-making in water management. Decision Support Systems 42, 2261-2273.

Srdjevic, B., Srdjevic, Z., Blagojevic, B., Suvocarev, K., (2013). A two-phase algorithm for consensus building in AHP-group decision making. Applied Mathematical Modelling 37, 6670-6682.

Sun, B.Z., Ma, W.M., (2015). An approach to consensus measurement of linguistic preference relations in multi-attribute group decision making and application. Omega 51, 83-92.

Susskind, L., McKearnen, S., Thomas-Lamar J., (1999). The Consensus Building Handbook: A Comprehensive Guide to Reaching Agreement. Sage Publications, Thousand Oaks.

Szmidt, E., Kacprzyk, J., (2003). A consensus-reaching process under intuitionistic fuzzy preference relations. International Journal of Intelligent Systems 18, 837-852.

Tanino, T., (1990). On group decision making under fuzzy preferences, In: J Kacprzyk and M Fedrizzi, Eds, Multiperson Decision Making Using Fuzzy Sets and Possibility Theory, Kluwer Academic Publishers, Dordrecht, 172-185.

Wallenius, J., Dyer, J.S., Fishburn, P.C., Steuer, R.E., Zionts, S., Deb, K., (2008). Multiple criteria decision making, multiattribute utility theory: recent accomplishments and what lies ahead. Management Science 54, 1336-1349.

Wu, J., Chiclana, F., Herrera-Viedma, E., (2015). Trust based consensus model for social network in an incomplete linguistic information context. Applied Soft Computing 35, 827-839.

Wu, J., Xiong, R., Chiclana, F., (2016). Uninorm trust propagation and aggregation methods for group decision making in social network with four tuple information. Knowledge-Based Systems 96, 29-39.

Wu, Z.B., Xu, J.P., (2016a). Possibility distribution-based approach for MAGDM with hesitant fuzzy linguistic information. IEEE Transactions on Cybernetics 46, 694-705.

Wu, Z.B., Xu, J.P., (2012). A consistency and consensus based decision support model for group decision making with multiplicative preference relations. Decision Support Systems 52, 757-767.

Wu, Z.B., Xu, J.P., (2016b). Managing consistency and consensus in group decision making with hesitant fuzzy linguistic preference relations. Omega 65, 28-40.

Xu, J.P., Wu, Z.B., (2013). A maximizing consensus approach for alternative selection based on uncertain linguistic preference relations. Computers \& Industrial Engineering 64, 999-1008.

Xu, J.P., Wu, Z.B., Zhang, Y., (2014). A consensus based method for multi-criteria group decision making under uncertain linguistic setting. Group Decision and Negotiation 23, 127-148. 
Xu, X.H., Du, Z.J., Chen, X.H., (2015a). Consensus model for multi-criteria large-group emergency decision making considering non-cooperative behaviors and minority opinions. Decision Support Systems 79, 150-160.

Xu, Y.J., Zhang, W.C., Wang, H.M., (2015b). A conflict-eliminating approach for emergency group decision of unconventional incidents. Knowledge-Based Systems 83, 92-104.

Yager, R.R., (1988). On ordered weighted averaging aggregation operators in multicriteria decision making. IEEE Transactions on Systems, Man and Cybernetics, Part A: Systems and Humans 18, 183-190.

Yager, R.R., (2008). Intelligent social network analysis using granular computing. International Journal of Intelligent Systems 23, 1197-1219.

Yager, R.R., (2002). Defending against strategic manipulation in uninorm-based multi-agent decision making. European Journal of Operational Research 141, 217-232.

Yager, R.R., (2001). Penalizing strategic preference manipulation in multi-agent decision making. IEEE Transactions on Fuzzy Systems 9, 393-403.

Yu, L., Lai, K.K., (2011). A distance-based group decision-making methodology for multi-person multi-criteria emergency decision support. Decision Support Systems 51, 307-315.

Zhang, G.Q., Dong, Y.C., Xu, YF., (2014). Consistency and consensus measures for linguistic preference relations based on distribution assessments. Information Fusion 17, 46-55.

Zhang, G.Q., Dong, Y.C., Xu, Y.F., Li, H.Y., (2011). Minimum-cost consensus models under aggregation operators. IEEE Transactions on Systems, Man and Cybernetics, Part A: Systems and Humans 41, 1253-1261.

Zhang, H.J., Dong, Y.C., Chen, X., (2017a). The 2-rank consensus reaching model in the multigranular linguistic multiple-attribute group decision-making. IEEE Transactions on Systems, Man, and Cybernetics: Systems, in press, doi: 101109/TSMC20172694429.

Zhang, Z., Guo, C.H., Martínez, L., (2017b). Managing multi-granular linguistic distribution assessments in large-scale multi-attribute group decision making. IEEE Transactions on Systems, Man, and Cybernetics: Systems 47, 3063-3076.

Zhang, Z., Guo, C.H., (2016). Consistency and consensus models for group decision-making with uncertain 2-tuple linguistic preference relations. International Journal of Systems Science 47, 2572-2587.

Zhang, B.W., Liang, H.M., Gao, Y., Zhang, G.Q., (2018). The optimization-based aggregation and consensus with minimum-cost in group decision making under incomplete linguistic distribution context. Knowledge-based systems, in press, doi: 10.1016/j.knosys.2018.05.038. 